\title{
A systematic review and meta-analysis of the impact of collaborative practice between community pharmacist and general practitioner on asthma management
}

This article was published in the following Dove Press journal:

Journal of Asthma and Allergy

\author{
Naeem Mubarak ${ }^{1,2}$ \\ Ernieda Hatah ${ }^{3}$ \\ Tahir Mehmood Khan ${ }^{4}$ \\ Che Suraya Zin' \\ 'Kulliyyah of Pharmacy, Department of \\ Pharmacy Practice, International Islamic \\ University, Kuantan, Malaysia; ${ }^{2}$ Lahore \\ Pharmacy College, Lahore Medical \& Dental \\ College, University of Health Sciences, \\ Lahore, Pakistan; ${ }^{3}$ Faculty of Pharmacy, \\ University Kebangsaan Malaysia, Kuala \\ Lumpur, Malaysia; ${ }^{4}$ Institute of \\ Pharmaceutical Sciences, University of \\ Veterinary and Animal Sciences, Lahore, \\ Pakistan
}

Objective: This systematic review aims to investigate the impact of collaborative practice between community pharmacist (CP) and general practitioner (GP) in asthma management. Methods: A systematic search was performed across 10 databases (PubMed, Medline/Ovid, CINAHL, Scopus, Web of Science, Cochrane central register of controlled trials, PsycARTICLES ${ }^{\circledR}$, Science Direct, Education Resource Information Centre, PRO-Quest), and grey literature using selected MeSH and key words, such as "community pharmacist", "general practitioner", and "medicine use review". The risk of bias of the included studies was assessed by Cochrane risk of bias tool. All studies reporting any of the clinical, humanistic, and economical outcomes using collaborative practice between CPs and GPs in management of asthma, such as CPs conducting medications reviews, patient referrals or providing education and counseling, were included.

Results: A total of 23 studies (six RCTs, four C-RCT, three controlled interventions, seven prepost, and three case control) were included. In total, 11/14 outcomes were concluded in favor of CP-GP collaborative interventions with different magnitude of effect size. Outcomes, such as asthma severity, asthma control, asthma symptoms, PEFR, SABA usage, hospital visit, adherence, and quality of life (QoL) (Asthma Quality-of-Life Questionnaire [AQLQ]; Living with Asthma Questionnaire [LWAQ]) demonstrated a small effect size $(\mathrm{d} \geq 0.2)$, while inhalation technique, ED visit, and asthma knowledge witnessed medium effect sizes (ES) $(d \geq 0.5)$. In addition to that, inhalation technique yielded large ES $(\mathrm{d} \geq 0.8)$ in RCTs subgroup analysis. However, three outcomes, FEV, corticosteroids usage, and preventer-to-reliever ratio, did not hold significant ES $(\mathrm{d}<0.2)$ and, thus, remain inconclusive. The collaboration was shown to be value for money in the economic studies in narrative synthesis, however, the limited number of studies hinder pooling of data in meta-analysis.

Conclusion: The findings from this review established a comprehensive evidence base in support of the positive impact of collaborative practice between CP and GP in the management of asthma. Keywords: community pharmacist, general practitioner, inter-professional collaboration, asthma, collaborative care, clinical outcomes

\section{Introduction}

Among the four major groups of chronic diseases, chronic respiratory diseases have the second highest estimated economic burden for 2011-2025 (US\$ 1.59 trillion) and are responsible for $15 \%$ of deaths in the world. ${ }^{1}$ Chronic respiratory diseases affect air passages and associated structures of lungs, which lead to either airways'
Lahore Pharmacy College, Lahore Medical \& Dental College, University of Health Sciences, Lahore, North Tulspura, Canal Bank Road, Lahore 54000, Pakistan Tel +923335553729

Email naeem.mubarak@lmdc.edu.pk 
obstruction or restriction. Examples of chronic respiratory diseases, which are in the headlines for global mortality and morbidity, include asthma, chronic obstructive pulmonary disease, pulmonary hypertension, and occupational lung disease.

Asthma is defined as a "heterogenous disease, usually characterized by chronic airway inflammation. It is defined by the history of respiratory symptoms, such as wheeze, shortness of breath, chest tightness and cough that vary over time and in intensity, together with variable expiratory airflow limitation". ${ }^{2}$ In low- and middle-income countries, asthma is comparatively more pervasive than any other chronic respiratory disease, and is a prime mover of mortality and disability in all age and gender groups, especially children. In 2015 alone, the death toll due to asthma was 383,000 globally. ${ }^{1,3,4}$

It is recommended that every country should make updated strategies for efficient diagnosis and medicine management of asthma with an emphasis on capacity building of health professionals. The capacity building may include reinforcing the role of potential healthcare professionals and strengthening the integration of community and primary healthcare by finding innovative ways and new horizons for collaborative efforts and actions. ${ }^{1,2,5,6}$ Effective management of asthma heavily depends on the strategy of medication management to improve adherence and to avoid any medication misadventure. This management of medication is essential, since the patient is taking medications long-term on a daily basis and there are many groups of medications involved in asthma management, such as short acting beta agonist (SABA), long acting beta agonist (LABA), and inhaled steroids.

\section{Involvement of general practitioner in management of asthma}

General practitioners (GPs) have generally been involved in asthma management in primary care. However, some studies reported significant shortfalls of GPs' practices to adhere to the standard guidelines and in commissioning written action plans for asthmatic patients, resulting in suboptimal clinical and humanistic outcomes. ${ }^{7,8}$ GPs, however, defend their positions by recounting many barriers in optimization of chronic disease management for asthmatics, for instance, high patient influx, fast growing aging populations, time constraints, and tough schedules, which makes it practically impossible to cater individual detailing and individualized care plans. On the other hand, patients lack motivation or proper understanding, and do not follow routine scheduled visits due to, for example, financial constraints, and only rush to GPs in the case of medical emergency, such as an acute asthma exacerbation. Together, these factors may contribute to stall effective management of asthma and, hence, call for sharing of responsibilities with other healthcare professionals by involving them in decision-making through a collaborative approach. ${ }^{9,10}$

\section{Involvement of community pharmacist in management of asthma}

The philosophy of pharmaceutical care urges the pharmacist to take responsibility of direct patient care and to improve clinical, humanistic, and economic outcomes, especially in chronic diseases. Community pharmacists (CPs) represent a conspicuous part of pharmacist fraternity, who are equally trained, thoroughly skilled, and well qualified. Recently in developed countries, CPs have been involved in direct patient care for health promotion and medication management services in various chronic diseases, such as hypertension, diabetes, and asthma. ${ }^{11}$ The expanded role of CPs in patient's education, adherence support, and medication therapy management becomes more important as asthma involves a chronic cycle, specialized devices, periodic assessments, self-monitoring, and an individualized therapeutic plan, and CPs have vast potential to provide these interventions. ${ }^{12,13}$

\section{Collaboration between CP and GP}

Diseases management approaches are receiving volumes of interest in developed countries as a valuable tool to combat with the burden of chronic diseases. A disease management approach is generally comprised of multiple components, such as medication management, education, counseling, monitoring, and use of appropriate guidelines. These approaches, no matters diverse in scope of practice, are based on a common underneath philosophy of "collaboration" among various healthcare stakeholders, such as pharmacists, physicians, and nurses, to deliver a wellcoordinated form of collaborative care. Medicines are the major key players in the management of chronic diseases. To improve quality and adherence in the medicine use process and to minimize the risk of any misadventure which chronic use of medicine may cause, medicine management programs were embedded as essential components in diseases management approaches. ${ }^{14}$ 
Collaborative practice such as "Physician-Pharmacist Collaborative Management" or "Collaborative Drug Therapy Management" which involves CPs may offer a peer review of the medication use process and ensure prescribed medications are in accordance to patient disease state. This concept, which was previously confined to tertiary healthcare, are now being practiced in primary healthcare settings such as community pharmacy. ${ }^{15}$ However, collaborative practices which involve $\mathrm{CP}$ and GP in primary care were less reported. CPs, as being situated at a foremost point of contact with patients, represent a consequential role for relationship building between patients and care-givers. A recent study in the US reveals approximately 250 million people enter pharmacies in a week, making it a unique premises in the sense of high ease of public access. ${ }^{16}$ Collaboration in the healthcare system involves mutual communication and sharing of expertise, knowledge, and experience of healthcare professionals, with a prime objective to benefit the patients. ${ }^{17}$ Hence, any model which involves two or more healthcare professionals (in this review GP and $\mathrm{CP}$ ) to improve patient outcomes can be considered as "collaborative practice". The practice may include, but not be limited to:

(a) training each other for a proposed intervention,

(b) referral of patients to each other for diseases or medication counseling or education,

(c) updating each other about patient drug usage patterns with or without any recommendation, and

(d) exchange of recommendations, such as change in the medication dose, dosage form, or medicine/ drug use review outcomes, or consultation about the course or state of the disease, done by using any form of communication, such as verbal, electronic, or written.

\section{Problem statement}

\section{Suboptimal management and burden of asthma}

Management of asthma remains suboptimal and represents a global health problem which has raised many concerns to countries' healthcare systems. ${ }^{1,2,5,6}$ Today, if we appraise the burden of asthma there is much to be worried about. Asthma stands out of the crowed as one of the most high-priced chronic diseases in the world. Economic studies have suggested that a poorly controlled asthma poses a far higher economic burden than a well-controlled asthma. ${ }^{18}$ The Global Asthma Report-2018 revealed the rampant asthma burden, which has amplified from 235 million cases in 2011 to 339 million cases in 2018 around the globe. Physical and psychosocial factors associated with asthma may reduce an individual's quality-of-life (QoL), causing disabilities, loss of productivity (particularly for pediatrics), deaths, and economic drain. The avoidable deaths related to asthma are still on a surge, with the utmost reason proclaimed to be the mismanagement of diseases and medications including "over-reliance on reliever medication rather than preventer medication".

\section{Lack of adherence and patient education}

Lack of adherence to medications, poor knowledge of inhaler technique, triggers, and disease are major contributors in the inadequate management of asthma. ${ }^{19}$ Basically, it is pertinent to mention that much of the burden is avoidable, and strategies are required to improve the management of asthma. A recent study in Europe concluded that only $5.3 \%$ of the asthma population met the defined clinical goals, and $75 \%$ of patients were nonadherent to the prescribed medications. This poor adherence in asthma is generally either linked to underuse of inhaled steroids or improper inhalation techniques, both of which are associated with poor asthma control. ${ }^{20}$ Apart from medication, it is of paramount importance that patients must understand what triggers asthma, how to avoid such triggers, and what to do in the case of an acute attack. In the US, the National Asthma Education and Prevention program has made formal patient education a mandatory component integrated in all kinds of asthma care programs. Thus, proper education and counseling of patients about the disease, medicines, and devices can lead asthmatics to enjoy a better QoL, and may reduce the burden of preventable emergency visits and deaths. ${ }^{21}$

\section{Research question}

The research was guided by a specific research question: "What is the impact of collaborative CP-GP interventions on clinical, humanistic, and economical outcomes in asthma patients?"

\section{Rationale of this systematic review}

Previous systematic reviews had evaluated the role of CPs in asthma management. However, many of them were not conclusive due to the lack of quantitative synthesis (metaanalysis) of the outcomes, ${ }^{10,19,21,22}$ while other reviews mix a different healthcare setting (hospital, or clinics, or community pharmacy), and thus lack specificity for 
community pharmacist, ${ }^{10,22-24}$ nevertheless one review focused on only one outcome, ie, adherence and attempted meta-analysis but again used A mixed setting and does not focus on collaboration. ${ }^{24}$

There is limited evidence documented in the literature for the effectiveness of CP-GP collaborative approach in asthma management in primary care. ${ }^{25}$ It is not known whether these interventions have positive, negative, or no effect on patient outcomes. Furthermore, to enrich the understanding of the effectiveness of an intervention, it has been a bench mark in scientific queries to encompass a multidimensional or holistic view of outcomes of an intervention for instance, humanistic or economic outcomes, rather than just focusing on therapeutic outcome. Hence, these rationales and gaps in the evidence necessitate a systematic review focused on CP-GP collaborative intervention for asthma management in terms of clinical, humanistic, and economical outcomes.

The present review takes an account of all three outcomes: clinical, humanistic, and economic, and, thus, has wide outcome coverage, yet, is specific to pool up the contribution of only community pharmacist intervention which were conducted in collaboration with GPs. It has attempted to quantitatively combine data where it was combinable for each outcome in the form of meta-analysis. It is pertinent to mention the essential difference we want to clarify here is the distinction of the interventions as "collaborative" as compared to those where there is no collaboration (interventions administered solo, ie, independent of other healthcare provider), for example, smoking cessations programs offered through community pharmacy or asthma clinic run by independent pharmacists, because, sooner or later, these solo (independent) modes would proffer similar problems (lack of time for proper patient education and counseling, no peer review process of prescriptions) as reported by the critiques of physician's solo mode of care delivery, since GPs have been engaged independently in healthcare delivery for ages.

To date, there is no study, to our knowledge, that has systematically reviewed the outcomes of collaborative practice between GPs and CPs in the management of asthma. This expanded role of CPs and its collaboration with GPs needs an objective analysis that could lead to evidence which may sum up clinical, humanistic, or economic benefits theses collaborative practices may bring. Hence, the focus of this systematic review is asthma management strategies, which involve CPs interventions in collaboration with GPs. The aim of this systematic review is to summarize the evidence regarding the impact of collaborative care provided by CP-GP in management of asthma. Based on the findings of this review, our objective is to relate and connect various insights to put forward some recommendations to inform the policymakers about the impact of this collaborative care in the management of asthma. Consequently, this evidence base would be placed in the context of the Malaysian healthcare system to explore a number of evidence-based policy options.

\section{Methods}

\section{Protocol}

The protocol for this review was registered on the "International Prospective Register of Systematic Reviews" (record CRD42017057188). This systematic review was reported in accordance with the PRISMA guidelines. ${ }^{26}$ Thus, the research question was broken up into Population, Intervention, Outcome, Timings, and Settings (PICO-TS), in Table 1, explaining the pre-defined inclusion and exclusion criteria in detail. The review team ensures the validity of the systematic review, where NM was the main reviewer, CSZ the supervisor, EH the co-supervisor, and TMK an expert of meta-analysis.

\section{Search strategy}

Articles were located in 10 databases: NLM PubMed, Medline/Ovid, Cumulative index to Nursing and Allied Health Literature (CINAHL), Scopus, Web of Science, Cochran central register of controlled trials, PsycARTICLES $®$, Science Direct, Education Resource Information Centre, and PRO-Quest, from inception to January 1, 2017 using the search terms and Medical Subject Heading (MeSH) determined prior to the study using input from experience researchers, librarians, databases specific thesaurus, keywords, and MeSH used in relevant literature. Examples of search terms and $\mathrm{MeSH}$ used were: "pharmacist", "community pharmacy", "retail pharmacy", "general physician", "general practitioner", "medicine use review", "drug use review", and "medication therapy management". Search terms were constructed in accordance with the PICO model to build a fully fledged search strategy. As databases have differences in terms of search operations and MeSH words, so we tailored our main search strategy as per the requirements of an individual database. Full keywords and MeSH used for locating relevant articles in different databases are available in Appendix I. Boolean logic, wildcards, truncations, 


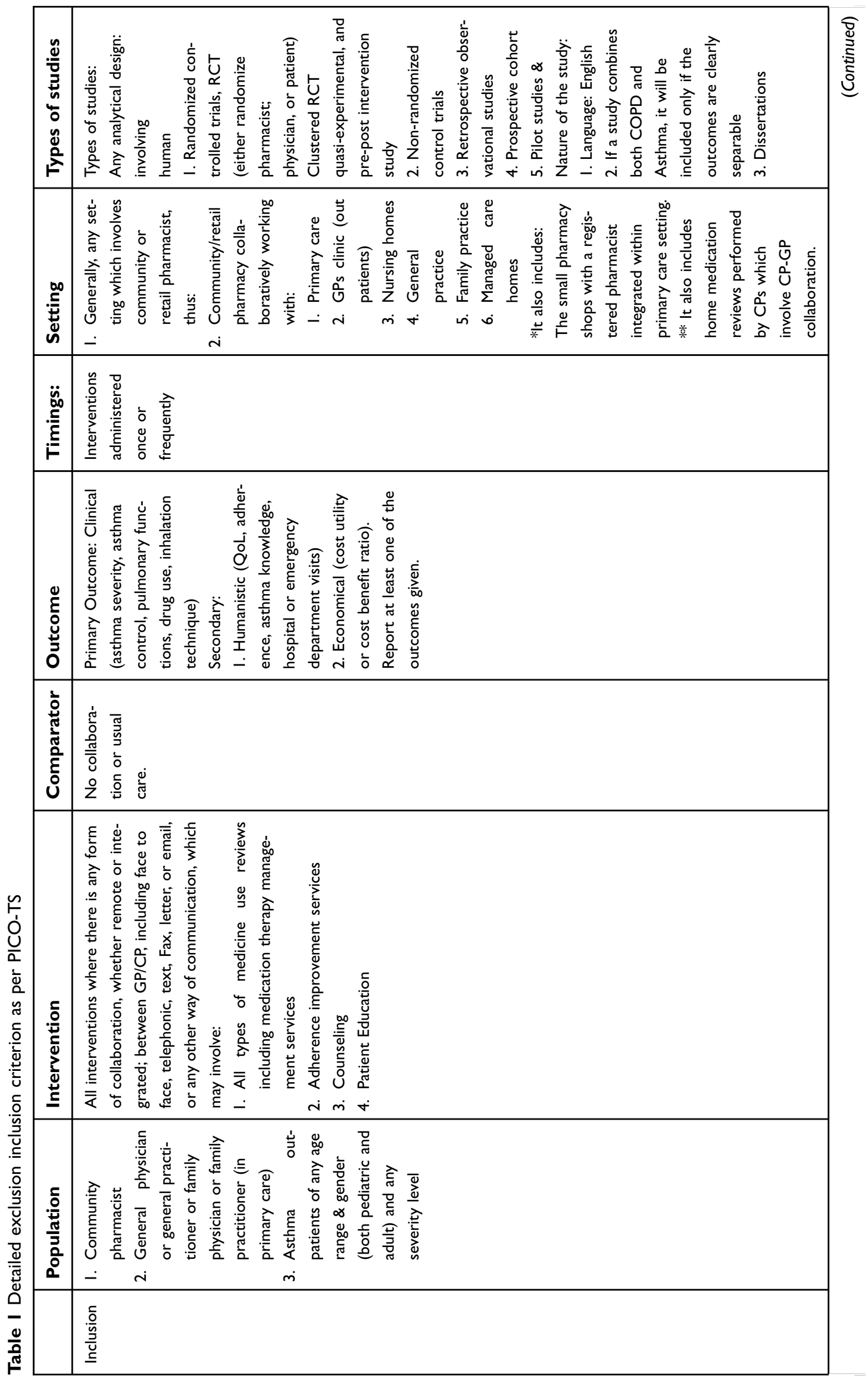




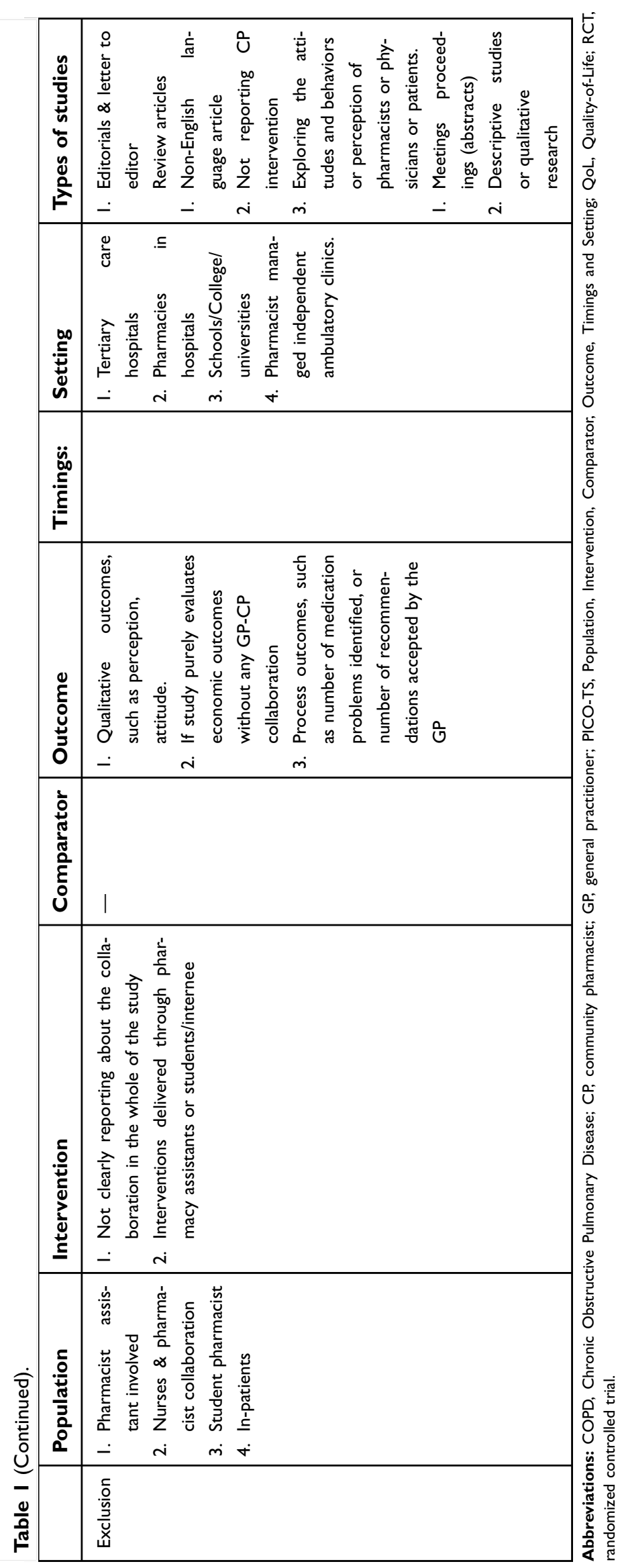


quotation marks, and proximity operators were used where applicable to reach an appropriate number of hits. A hand search of potentially relevant articles was then executed using the reference lists of relevant studies.

\section{Study selection}

Studies were identified through the search in electronic databases, grey literature search, and reference list of relevant articles. The retrieved articles were then de-duplicated in Endnotes, based on inclusion/exclusion criteria, and were subjected to title and abstract screening for eligibility for full text review by NM and checked by CSZ and EH. Whenever there was a doubt on the eligibility of an abstract, its full text was consulted. To be eligible, a study needs to have reported:

(a) An intervention performed by CPs for asthma management.

(b) Some degree of collaboration with a GP.

(c) Either a control group or pre-post research design.

(d) At least one of the three patient outcomes (clinical, humanistic, economical).

(e) Be in English language and not a conference proceeding.

Although randomized controlled trials (RCTs) are taken as gold standard in evidence synthesis, it is also a valid assumption to consider that RCT design is not feasible in all situations, so we added controlled trials without randomization as well as other quasi experimental research designs. A study was included if it complied with the pre-set inclusion and exclusion criteria. The examples of collaborative intervention include patient referral, prescription review, education, and counseling, where the comparator was usual care (where no $\mathrm{CP} / \mathrm{GP}$ collaboration is involved) in a setting which involved community pharmacy and primary care. Detailed inclusion/exclusion criteria are outlined in Table 1 . To ensure the validity of the screening process, all excluded articles were reviewed by all authors. In the case of any disagreement on a study for inclusion or exclusion; resolution was achieved through consensus. In the case of any doubt on the nature of collaboration, the corresponding authors were contacted via email to get the larger picture. This wais then followed by data extraction of important information and analysis.

\section{Data extraction}

Full text studies were duly reviewed, and data were extracted by two members (NM and CSZ) independently to minimize the bias, using a common standardized template for data extraction. This template had columns to add: study characteristics, type of intervention (education, counseling, medicine use review, referral to GPs, inhalation technique improvements, medication adherence assessment, and asthma control), mode of intervention to the patients (face-to-face or through any mail mode), and nature of collaboration between CPs and GPs based on a hypothetical collaboration scale. Any difference between the two members in extracted data was identified and resolved through consensus. Throughout the process of data extraction, we trusted only the data published in the article.

\section{Outcome of interest}

Primary outcomes (clinical) include pulmonary functions (peak expiratory flow rate (PEFR), forced expiratory volume $1\left(\mathrm{FEV}_{1}\right), \mathrm{FEV}_{1}$ /forced vital capacity (FVC), inhalation technique, asthma control, and drug usage. Secondary outcomes include reported results on humanistic outcomes, such as QoL, asthma knowledge, and economic outcomes, for example cost-benefit ratio.

\section{Collaboration scale}

The authors designed an arbitrary hypothetical collaboration scale to subjectively evaluate the level of collaboration involved in the included studies. Studies were ranked in terms of the nature of collaborative practice involved. If a study mentioned training of CPs on the management of asthma by the GP or vice versa it had 1 point. On the other hand, if a study mentioned patient referral from GP to CP or vice versa, it was given 2 points; while, a study which highlighted the fact that CPs updated GPs about patient drug usage patterns or diseases state had 3 points and, finally, a study which stated that CPs had a role which allowed themo suggest change of drug or dose and GPs accepted the recommendation was given 4 points on the collaboration scale (the highest score). The maximum points a study could achieve on the collaboration scale were 10 (ie, if the study had reported all four forms of collaboration), where higher scores represent a higher level of collaboration. The collaboration was termed "integrated" if the GPs and CPs share the same premises or "remote" if they do not share the same premises and communicate via phone or emails. 


\section{Data analysis}

Cochrane Review Manager (RevMan) ${ }^{\circledR}$ V.5.3 software (Copenhagen: The Nordic Cochrane Centre, The Cochrane Collaboration, 2014) was used for data analysis.

\section{Risk of bias (RoB) assessment}

RevMan was used to generate the ROB graphically. We used different RoB tools for different research designs to appraise RoB in various studies. For instance, (RCT), Controlled trials (CT), and Cluster randomized control trials (C-RCT) were evaluated through the "Cochrane risk of bias tool" because oit is a validated tool for RCTs. However, the number of RCTs are limited, and thus allow addition of other research designs for which another tool by Cochrane, "Effective Practice and Organization of Care (EPOC)" was deployed for all nonrandomized, or other quasi experimental study designs (pre-post). In EPOC, the studies were evaluated against eight main domains: selection bias (randomization, concealment, baseline measures), baseline outcome difference, attrition bias, detection bias, contamination bias, and reporting bias. As per risk of bias domains, the studies were scored using low, high, and unclear risk. However, it is not encouraged to give some numerical value to the RoB, as per the new Cochrane guidelines, which focus on the domain-based analysis of the RoB in the included studies. $^{27}$

\section{Procedure of meta-analysis}

For meta-analysis, we grouped studies based on outcome and, where at least two studies report combinable data for a specific outcome, meta-analysis was carried out to pool the results. Thus, we performed independent meta-analysis for individual outcomes $(\mathrm{n}=14)$ contrasting CP-GP collaborator intervention vs usual care or no care.

As the inclusion criterion for study designs was general and allowed all types of research designs, the studies included in quantitative synthesis were partly different in terms of research designs and tools to measure outcomes. Thus, we combined the data, where it was possibly combinable and aided our findings through both; narrative synthesis (descriptive), as well as through meta-analysis. Only studies were pooled which have reported data in terms of mean and standard deviations (SDs) or in a form which can be convertible to mean or SD through some statistical operation. To compute effect size, standard mean difference was used for continuous variables, while odds ratio was used for categorical variables. Finally, to synthesize the summary effect estimate of CP-GP collaborative intervention, Forest plots were constructed based on the assumption of random effect model to pool the data for all outcome. A random effect model was deployed because it introspects various sources of variability or heterogeneity, ie, within and in between study variability due to sample size, research design, or tool used to measure the outcome of all the included studies. This is opposite of the fixed effect model, which only takes an account of the statistical heterogeneity among the included studies in meta-analysis. Furthermore, the random effect model yields a conservative effect size rather than an exaggerated one and, thus, does not produce false positive results. ${ }^{28}$ Three important considerations were taken into account before concluding a meta-analysis.

\section{Heterogeneity}

The most common misleading and malpractice while reporting meta-analysis is pooling of data when there is considerable heterogeneity, which gave rise to false conclusions. ${ }^{29-33}$ Thus, heterogeneity is a decisive factor to authorize whether the data in various studies are combinable or not. $\mathrm{Tau}^{2}$ or $\mathrm{chi}^{2}$ and $\mathrm{I}^{2}$ are some of the tests used to determine statistical heterogeneity, but the $\mathrm{I}^{2}$ does not suffer from some of the drawbacks of the other two tests, and thus holds more reliability. ${ }^{29} \mathrm{I}^{2}$ is the percentage of total variance. $\mathrm{I}^{2}$ values of $25 \%, 50 \%$, and $75 \%$ indicate low, moderate, and high heterogeneity, respectively. ${ }^{30}$ Thus, heterogeneity in the included studies in meta-analysis was accessed through $\mathrm{I}^{2}$ statistic, where a higher value of $\mathrm{I}^{2}$ means a higher level of variability because of factors other than random variations. An $\mathrm{I}^{2}$ value of zero or less than $25 \%$ gives credibility that the effect size produced by pooling of data of various studies represents the true value of a homogenous population (ie, the studies don't have different populations), and there is no need of subgroup or sensitivity analysis. ${ }^{30,34,35}$ On the other hand, high $\mathrm{I}^{2}$ value indicates that the populations across different studies in a meta-analysis are different, hence pooling of data makes no sense and should be avoided. In this case the next step is to perform a subgroup analysis to determine any valid reason of heterogeneity, if the heterogeneity value get insignificant in a subgroup analysis, then the pooled effect size of an intervention holds much credibility than the overall analysis. In our case, if $\mathrm{I}^{2}>25 \%$, we preferred to perform subgroup analysis based on research designs to get a subgroup which has low $\mathrm{I}^{2}$ values and 
may allow the pooling of data to draw valid results. Zlowodzki et al, ${ }^{36}$ suggested to determine the reasons of heterogeneity through subgroup analysis under a predefined criterion, and this review followed the guidelines provided for subgroup analysis. ${ }^{37}$

\section{Effect size (ES)}

"Effect" is a measure of association between an intervention or exposure and an outcome. Effect size is the magnitude or strength of the association, and is represented by "d", also called "Cohen's d". The beneficial or non-beneficial results of meta-analysis are indicated by effect size. ${ }^{38}$ Medical researchers are often interested to establish the superiority of one intervention over another. They do it by computing the difference between the intervention and control group. ${ }^{39}$

\section{Interpretation of ES}

As per Cohen's classification, an ES of magnitude 0.2, 0.5, and 0.8 denotes small, medium, and large ES, respectively. ${ }^{40}$ The effect size value may be positive or negative. For continuous outcomes, an ES of zero means there is no difference between the groups being compared, while, for binary outcomes the value of 1 would indicate there is no difference between the groups. ES has been discussed in details by Durlak, ${ }^{40}$ and throughout our metaanalysis we will stick to the interpretations and guidelines provided. $^{40,41}$

\section{Results}

After a careful title and abstract screening, 8,604 studies were excluded and 84 studies were examined in full text. Only 23 out of 84 studies were found at par with all the inclusion/exclusion criteria, while studies were excluded in full text screening because of different settings $(n=13)$, studies did not mention any level of CP-GP collaboration $(n=33)$, outcomes were not of interest $(n=10)$ or combined both chronic obstructive pulmonary disease and asthma $(\mathrm{n}=1)$, an ongoing study, ie, study protocols $(\mathrm{n}=3)$, and a descriptive study $(\mathrm{n}=1)$.

A total of 23 articles were included in the final review (Figure 1). There were six studies with RCT study designs, four C-RCT, three controlled interventions (CI), seven pre-post (PP) studies, and three case control studies (CC). Most of the studies were conducted in Australia $(n=8)$; followed by the US ( $n=3)$; UK, Germany, Canada $(n=2$ each), and Finland, Spain, Italy, Malta, Denmark, and Belgium ( $\mathrm{n}=1$ each).
There was a wide range of patient's age included in this review; age $\geq 18$ years $(n=18)$, age $\geq 12$ years $(n=4)$, and age $\geq 7$ years $(\mathrm{n}=1)$. The majority of the studies provided interventions to patients with moderate-to-severe asthma. The duration of the studies included in this review ranged from 6 weeks to 5 years; 6 weeks $(n=1), 3$ months $(n=1), 6$ months $(\mathrm{n}=11), 9$ months $(\mathrm{n}=2), 12$ months $(\mathrm{n}=7)$, and 5 years $(n=1)$. The most common mode of delivery of the asthma intervention was face to face $(\mathrm{n}=19)$, followed by "mailed intervention" $(\mathrm{n}=3)$. One study compared "mail vs face to face intervention", and one study used mix mode intervention ie, face to face and mail follow-up.

Seven studies reported the duration of the intervention provided by CPs per session. The duration of intervention, categorized by the researchers, was divided into: 10-30 minutes $(n=5), 31-44$ minutes $(n=1)$, and 45-60 minutes $(n=1)$. There was little information available on the duration of intervention in other studies $(n=15)$.

Furthermore, 14 studies reported the number of visits a patient had to attend $\mathrm{CP}$ during the study to receive intervention. The most common number of intended visits cited were four $(n=9)$, but it varies considerably based on the duration of study. As a matter of fact, the shorter the duration of the study the less visits can be possible. The most common type of CPs' intervention in these visits was patient education and counseling, which focused on: inhaler technique correction $(\mathrm{n}=10)$, asthma knowledge $(\mathrm{n}=11)$, self-management measures to keep asthma under control $(\mathrm{n}=9)$, drug use and therapy monitoring $(\mathrm{n}=9)$, and medication adherence $(\mathrm{n}=8)$. Nearly all studies employed more than one intervention.

The scores on the hypothetical collaboration scale showed that seven studies obtained scores of 10/10, while one study obtained $9 / 10$ and one study had an $8 / 10$ score. The remaining the studies $(n=9)$ have a score $<8 / 10$. "Patient referral" was the most frequent $(n=17)$ type of collaborative intervention cited in the studies, followed by "CP's training" ( $n=15)$, ie, CPs' training by physician, for a proposed intervention, such as on disease management or pathological basis asthma or how to respond to an emergency. Almost half of the studies $(n=11)$ mentioned the highest form of collaboration, ie, where CPs recommended their GPs for changes in medicine or dose. There was only one study which involved "integrated" collaborative practice between GP and $\mathrm{CP}$, while the rest of the studies $(n=22)$ involved remote collaboration.

A summary of the reported outcomes is provided in Table 2. Based on broad categories of outcomes as defined in the inclusion criteria (clinical, humanistic, and 


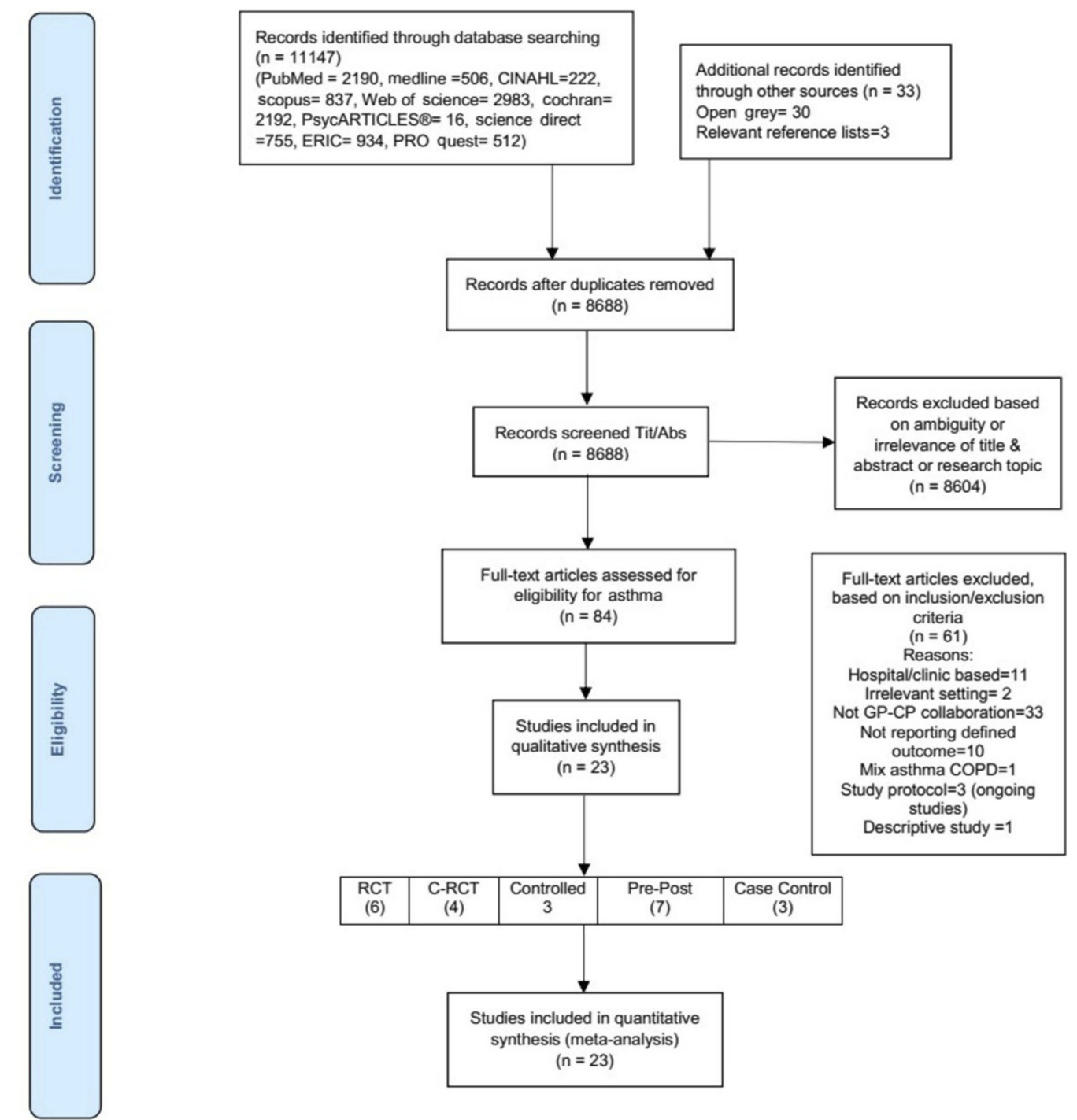

Figure I PRISMA flow diagram.

Abbreviations: COPD, chronic obstructive pulmonary disease; CP, community pharmacist; C-RCT, clustered randomized controlled trial; GP, general practitioner; RCT, randomized controlled trial; Tit/Abs, Title/Abstract; $n$, number.

economic outcomes), only four studies reported all three outcomes. On the other hand, five studies reported purely clinical outcomes and two studies reported only humanistic outcomes.

\section{Appraisal of risk of bias}

Summary graphs of Cochrane risk of bias and EPOC risk of bias are depicted in Figures 2-5, respectively. As an overall finding, the studies included were free of many types of biases. However, the most common bias present was the "blinding of personnel". However, this is understandable as in this kind of scenario where an educational intervention is administered to improve asthma knowledge or drug use process, blinding of either patient or personnel is not always possible.

\section{Impact of the collaborative interventions}

To pool up the results of outcomes, we opted both methods, ie, qualitative or narrative synthesis, as well as quantitative or meta-analysis by statistically combining (where possible) the results to produce a summary effect of individual outcomes.

\section{Evidence base through narrative synthesis}

To perform a narrative synthesis, outcomes were grouped into two broad categories:

(a) Favorable or significant (where the study clearly mentions a $p$-value less than 0.05 , indicating significant improvements by CP's intervention). 


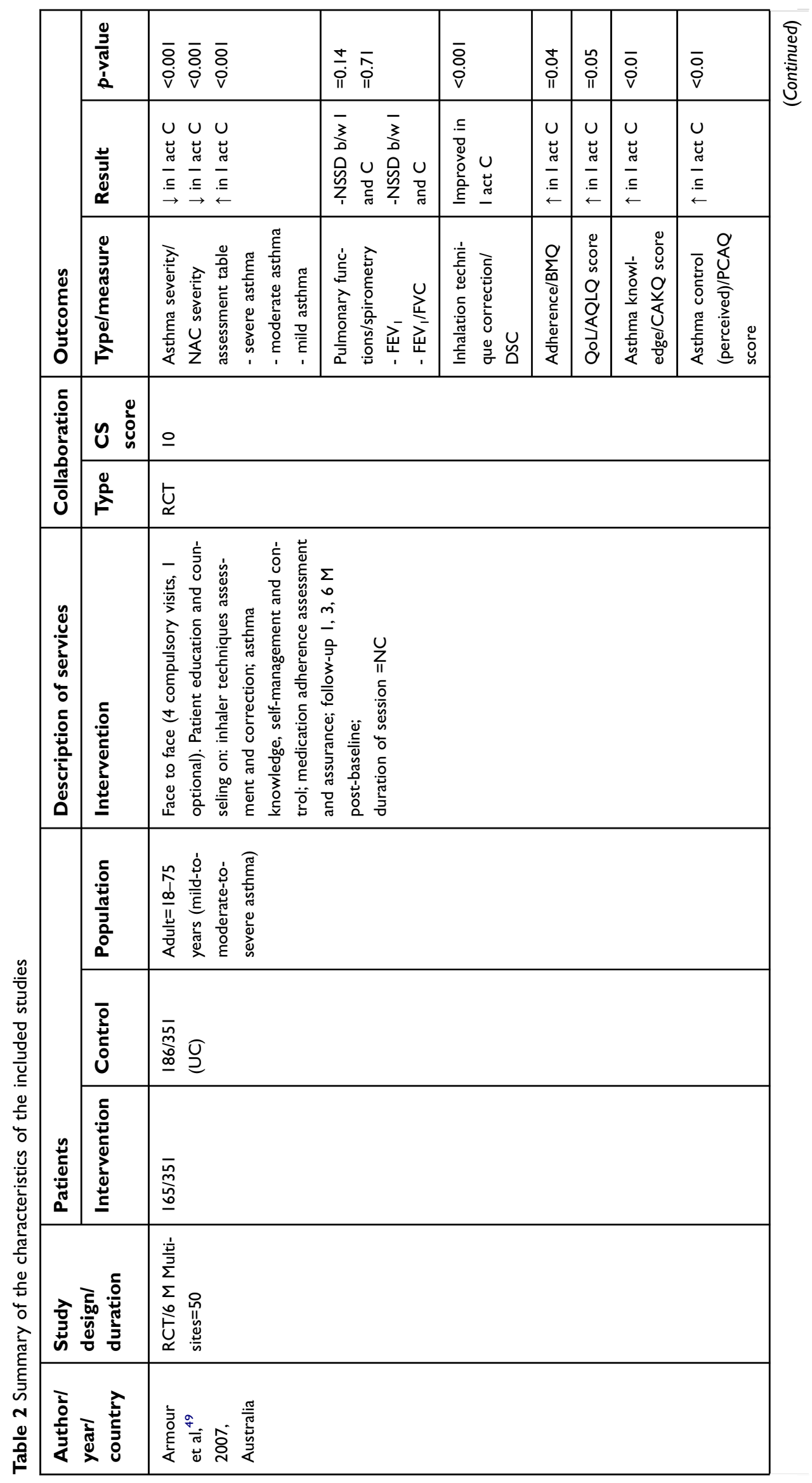




\begin{tabular}{|c|c|c|c|c|c|c|c|c|c|}
\hline \multirow[b]{3}{*}{ 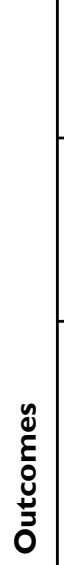 } & 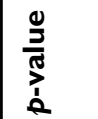 & 号 & $\begin{array}{l}\stackrel{2}{0} \\
\stackrel{0}{v}\end{array}$ & $\begin{array}{l}\overline{\mathrm{a}} \\
\mathrm{i}\end{array}$ & $\begin{array}{l}\overline{8} \\
\text { iv }\end{array}$ & $\overline{\dot{O}}$ & $\begin{array}{l}\overline{\mathrm{o}} \\
\mathrm{i}\end{array}$ & $\begin{array}{l}\overline{\text { o }} \\
\text { ì }\end{array}$ & $\begin{array}{l}\overline{8} \\
\dot{0}\end{array}$ \\
\hline & 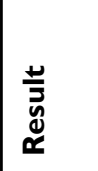 & 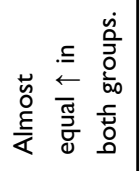 & 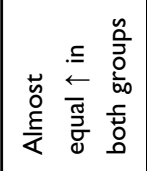 & 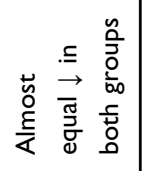 & 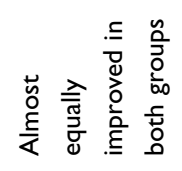 & 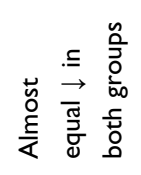 & 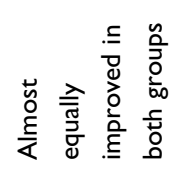 & 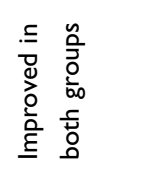 & 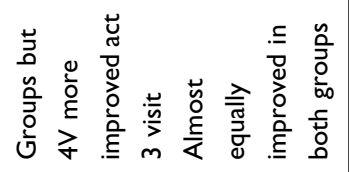 \\
\hline & 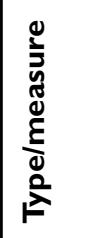 & 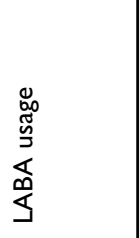 & 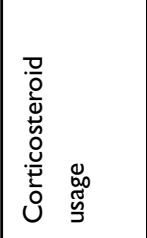 & 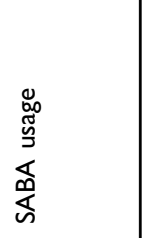 & 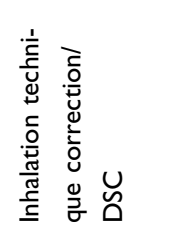 & 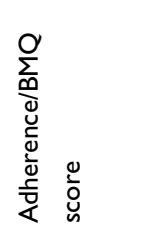 & 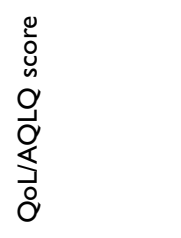 & 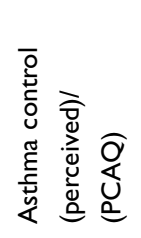 & 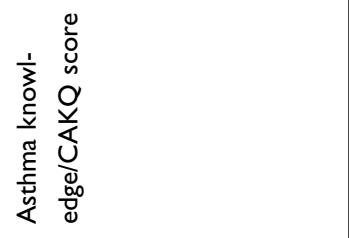 \\
\hline \multirow{2}{*}{ 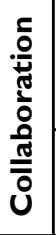 } & ป ํํํ & 으 & & & & & & & \\
\hline & $\stackrel{2}{2}$ & 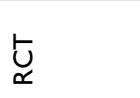 & & & & & & & \\
\hline 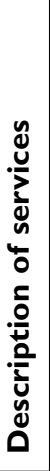 & 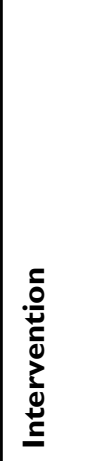 & 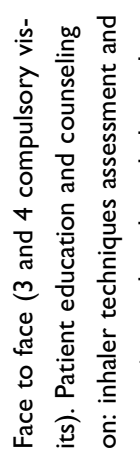 & 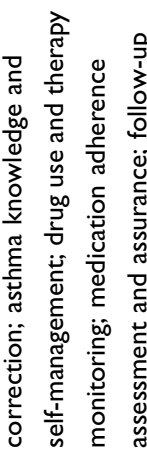 & 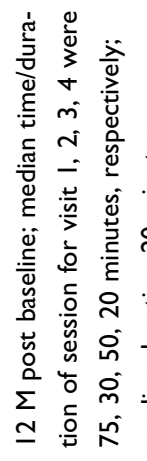 & & & & & \\
\hline \multirow[b]{3}{*}{ 营 } & $\begin{array}{l}\frac{0}{0} \\
\frac{0}{2} \\
\frac{0}{0} \\
0 \\
0\end{array}$ & 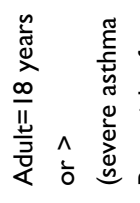 & 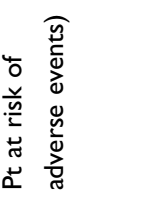 & & & & & & \\
\hline & 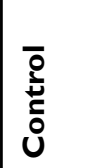 & 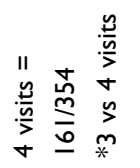 & 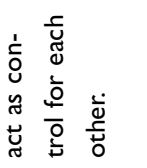 & & & & & & \\
\hline & 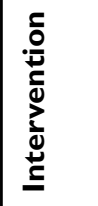 & $\begin{array}{l}\bar{m} \\
\frac{2}{11} \\
\frac{0}{n} \\
\frac{n}{2} \\
m \\
m\end{array}$ & & & & & & & \\
\hline \multicolumn{2}{|c|}{ 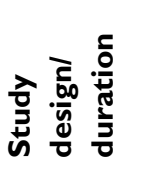 } & 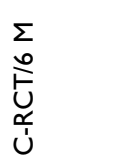 & & & & & & & \\
\hline \multicolumn{2}{|c|}{ 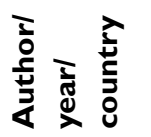 } & 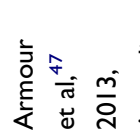 & & & & & & & \\
\hline
\end{tabular}




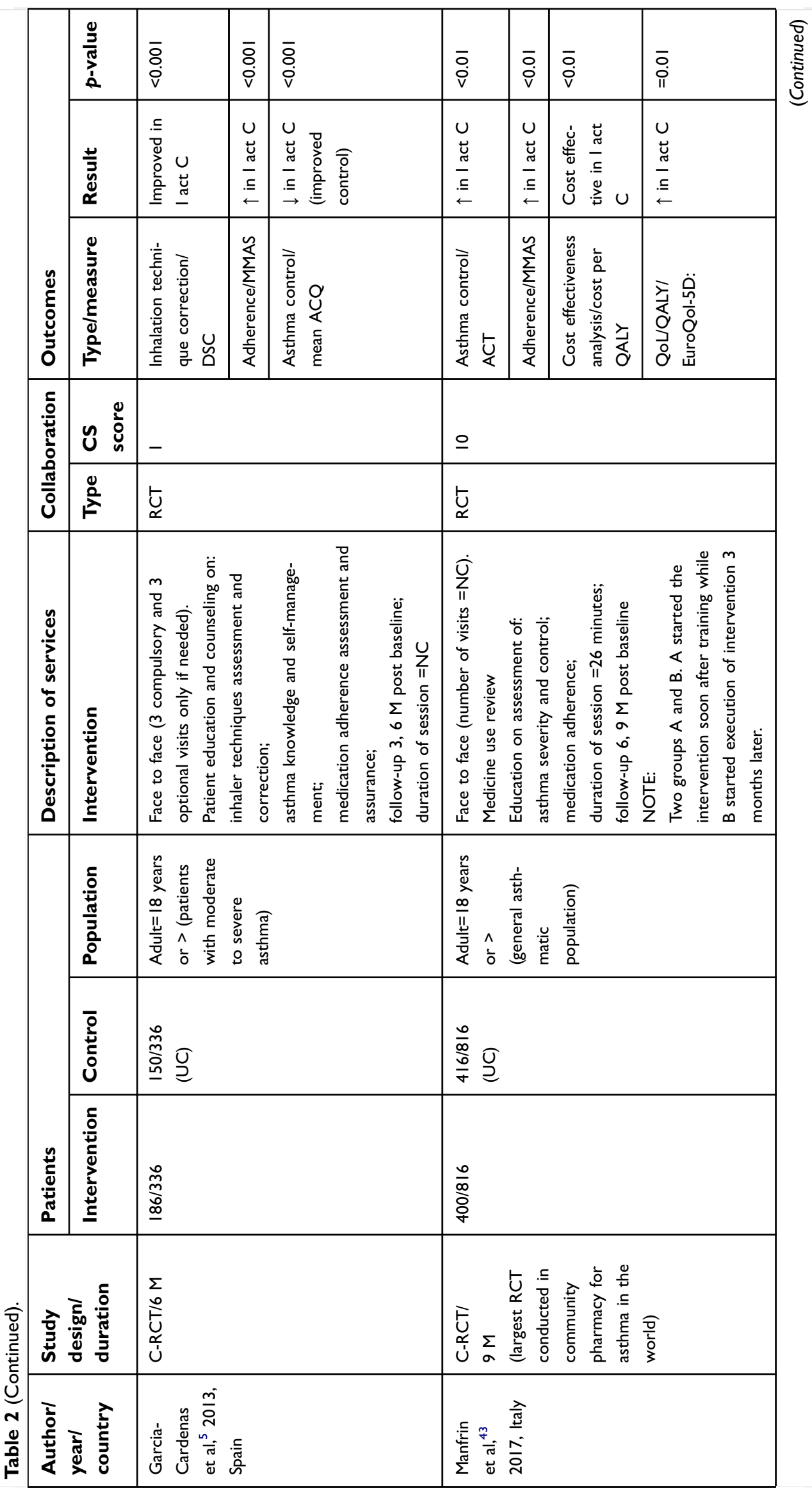




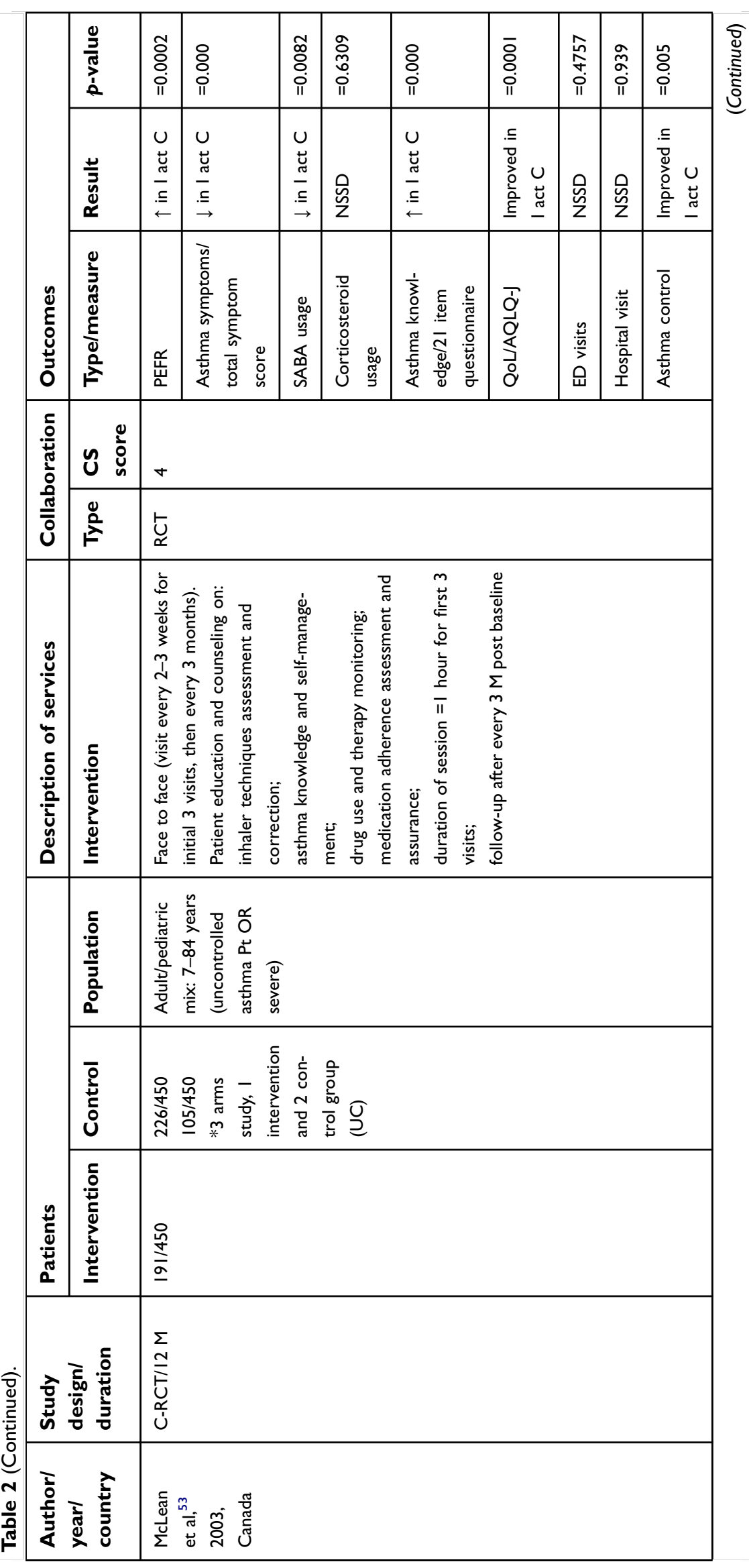




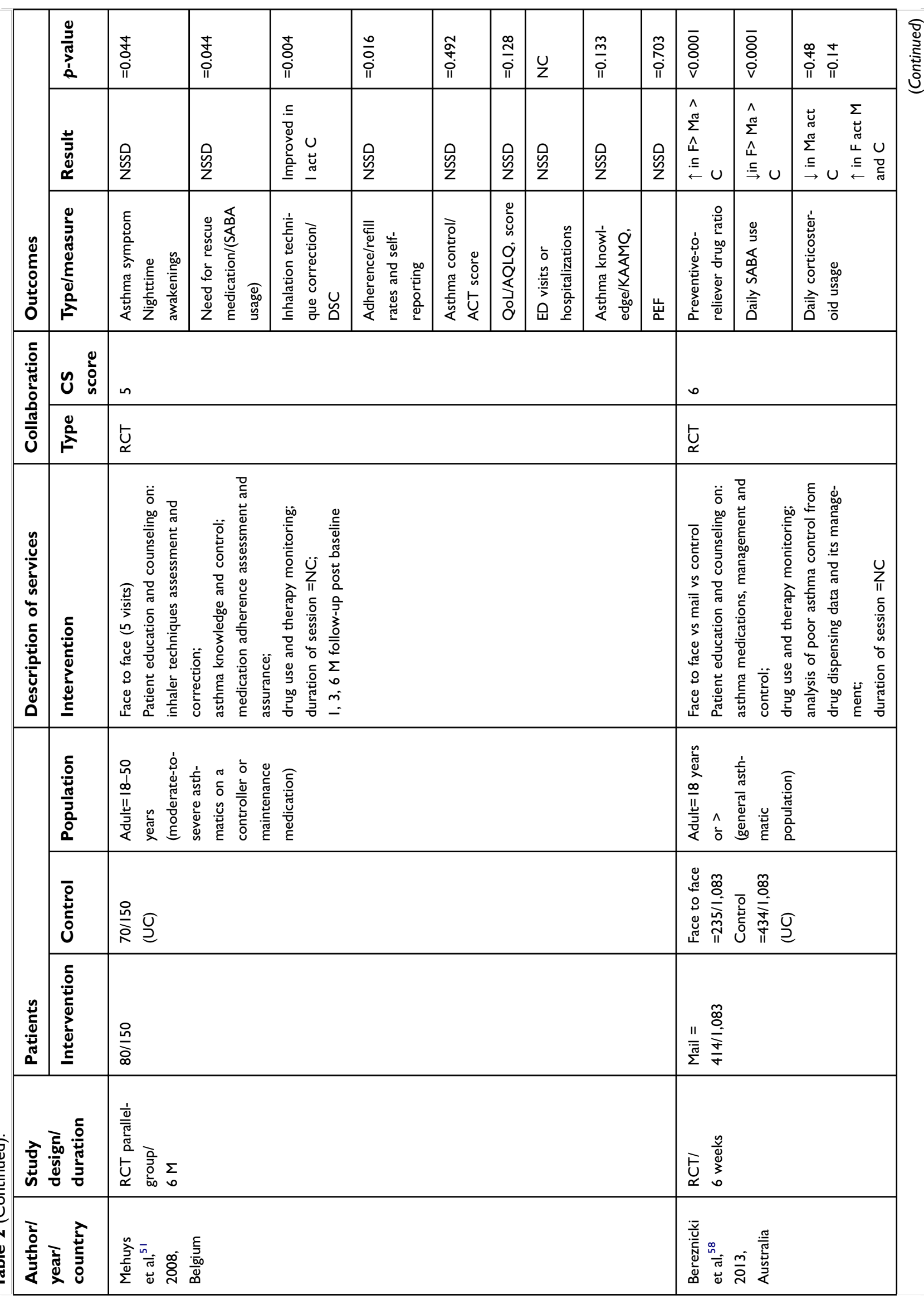




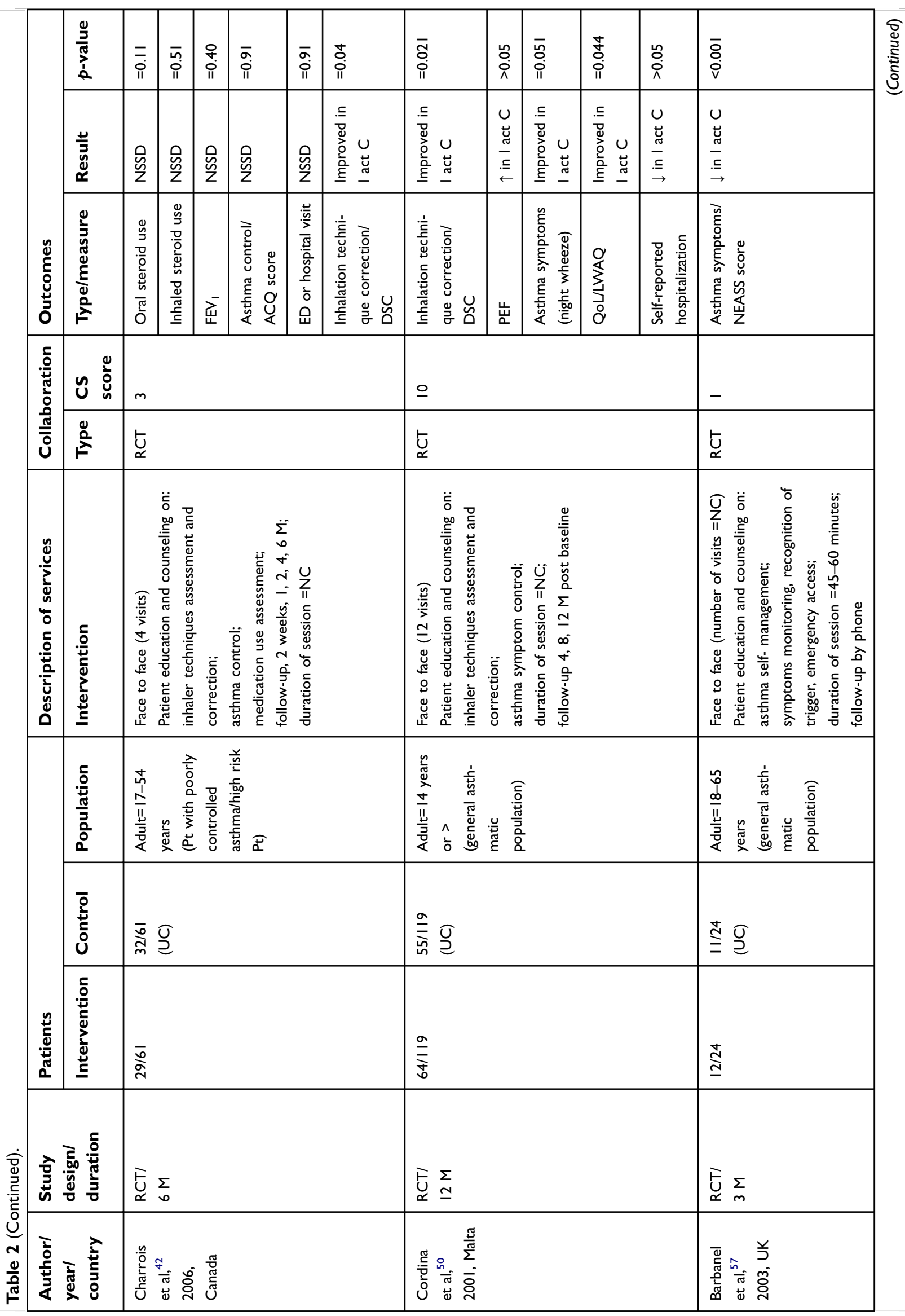




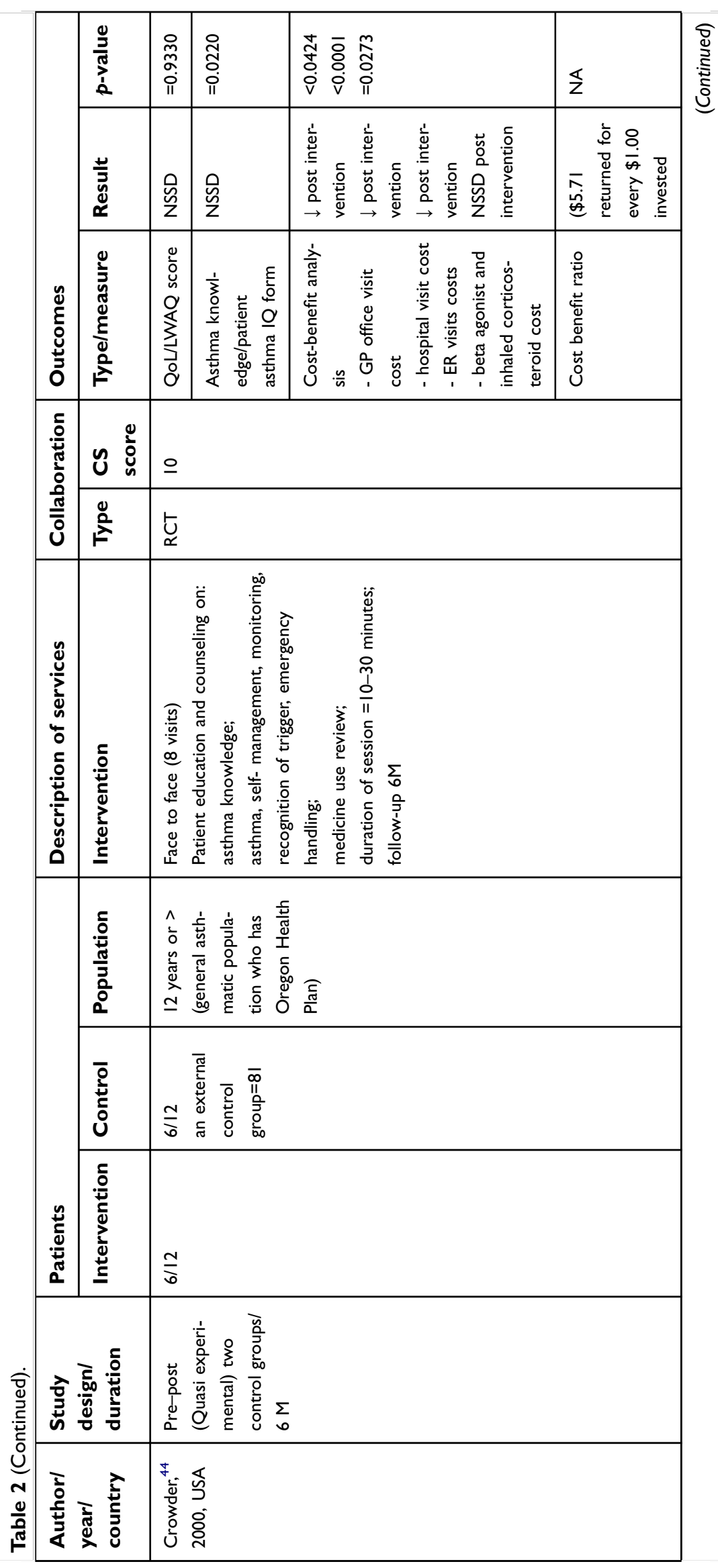




\begin{tabular}{|c|c|c|c|c|c|c|c|c|}
\hline & $\frac{0}{\sum_{0}^{0}}$ & $\begin{array}{l}\text { ö } \\
\text { o. } \\
\text { v }\end{array}$ & $\begin{array}{l}\overline{\bar{a}} \\
\text { oi } \\
\text { i }\end{array}$ & $\begin{array}{l}\bar{\partial} \\
\stackrel{0}{0} \\
\dot{i}\end{array}$ & סें & $\begin{array}{l}\text { o } \\
\stackrel{0}{0} \\
\text { v }\end{array}$ & $\underline{z}$ & $\underline{z}$ \\
\hline & 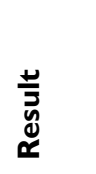 & & 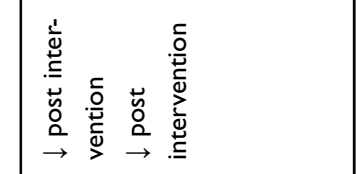 & 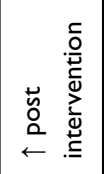 & 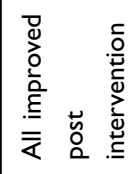 & 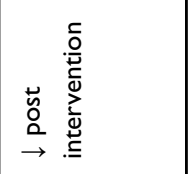 & 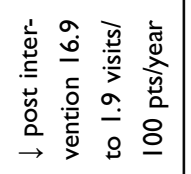 & 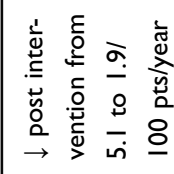 \\
\hline 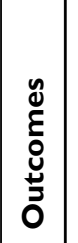 & 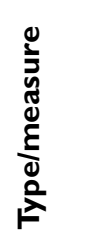 & & 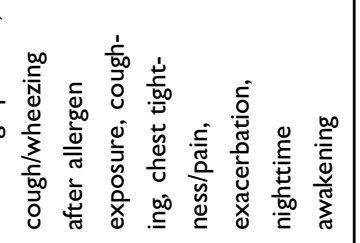 & 흘 & 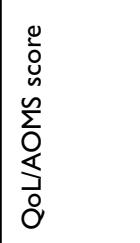 & 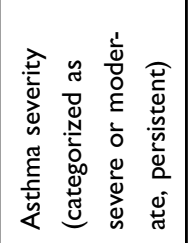 & $\begin{array}{l}\frac{n}{2} \\
\frac{n}{2} \\
\frac{8}{4}\end{array}$ & 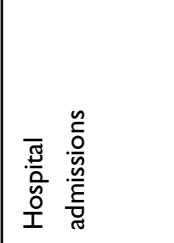 \\
\hline 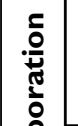 & uั & $\stackrel{\circ}{ }$ & & & & & & \\
\hline$\frac{\bar{c}}{\overline{0}}$ & 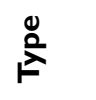 & $\underset{\propto}{\mathfrak{u}}$ & & & & & & \\
\hline 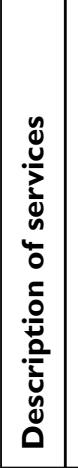 & 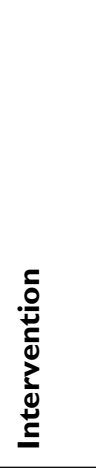 & & 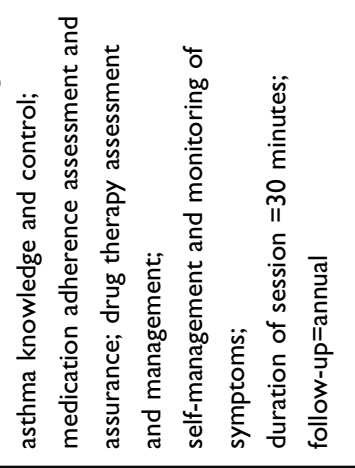 & & & & & \\
\hline & $\frac{2}{0}$ & & 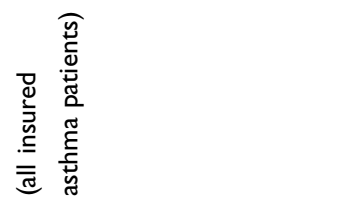 & & & & & \\
\hline & ن & $\mathbb{z}$ & & & & & & \\
\hline 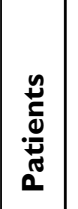 & & నิ & & & & & & \\
\hline & & & 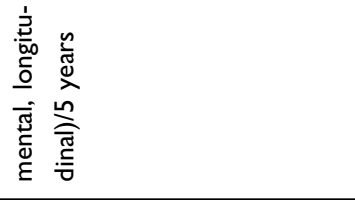 & & & & & \\
\hline 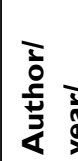 & 童 & & & & & & & \\
\hline
\end{tabular}




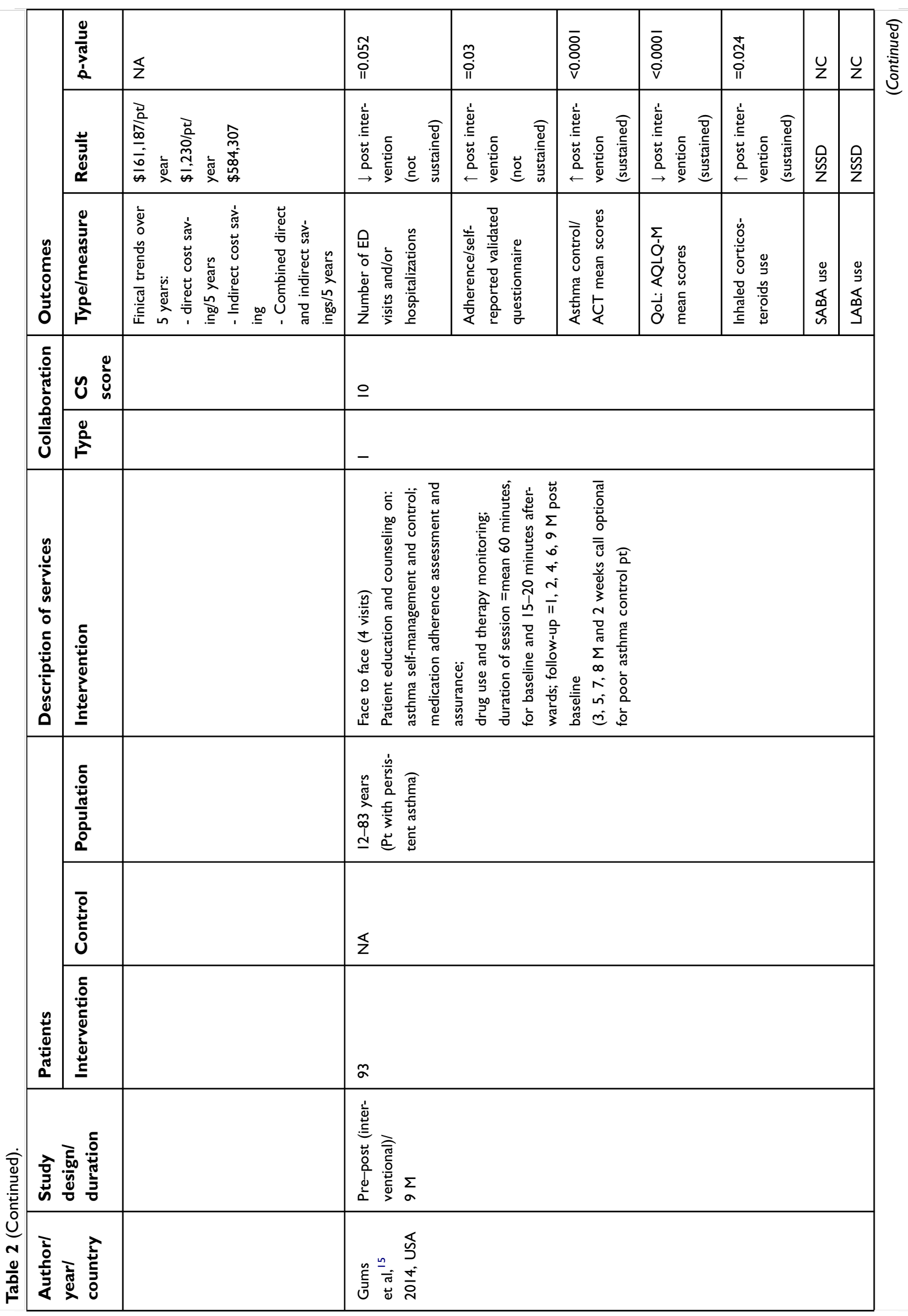




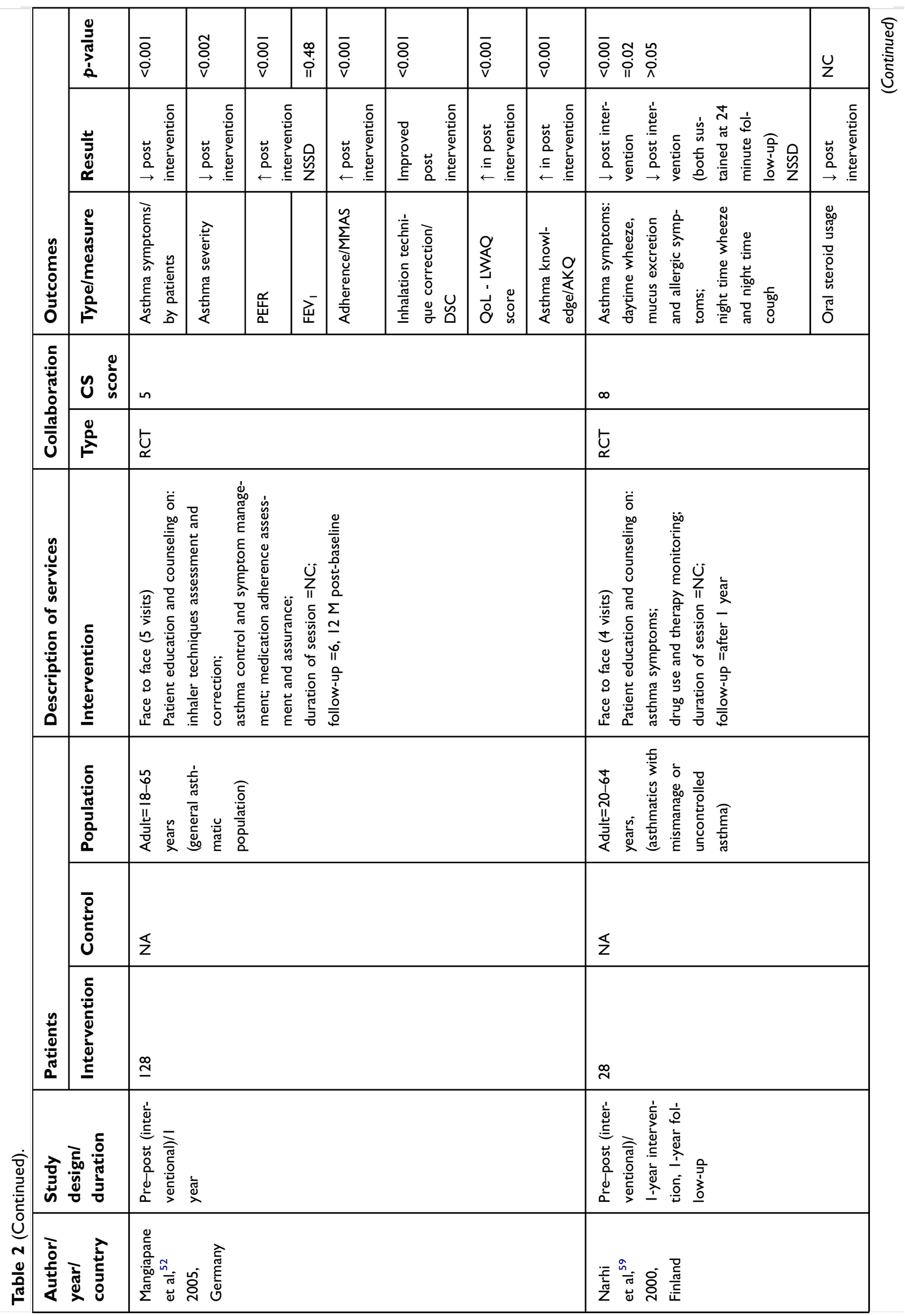




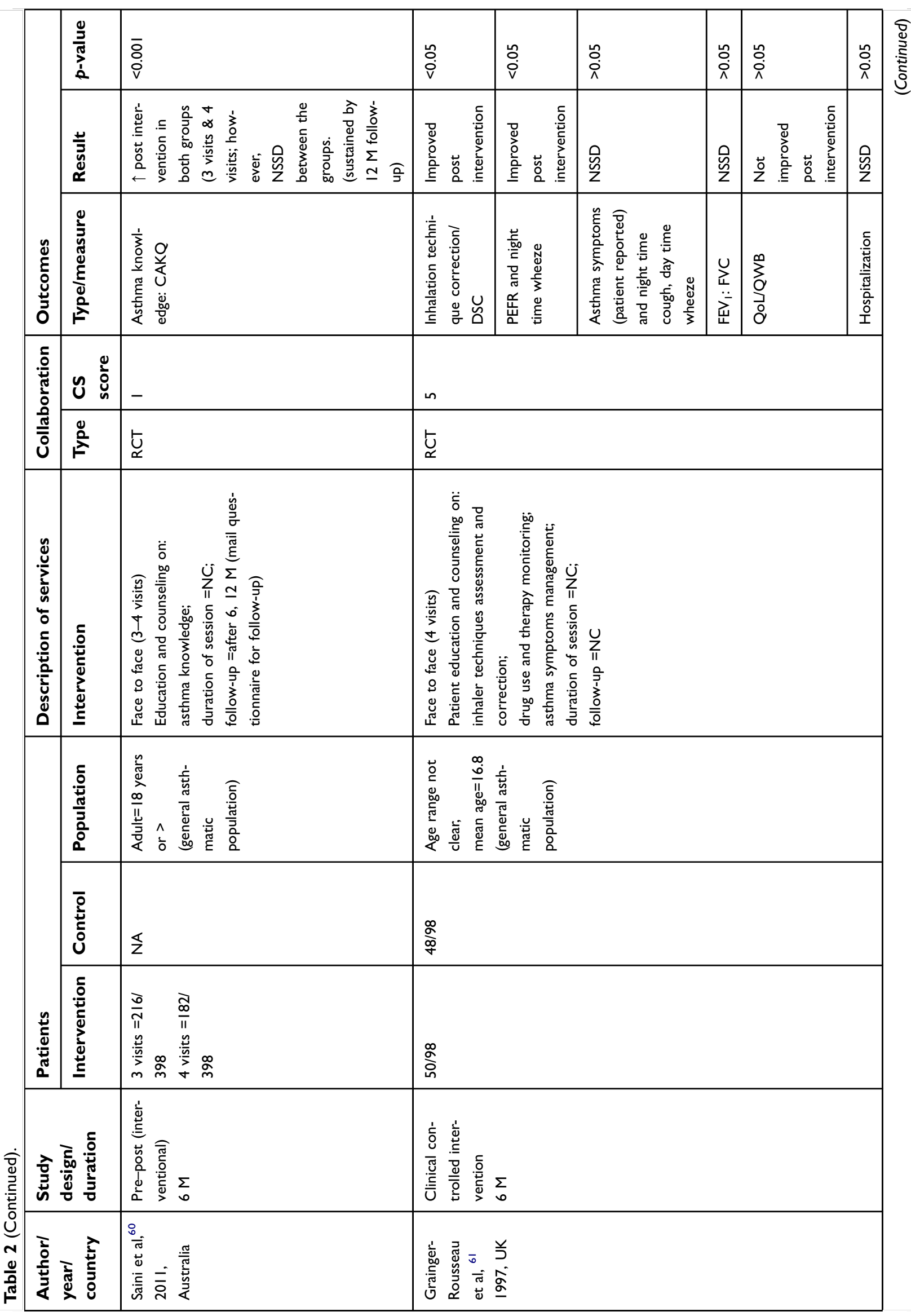




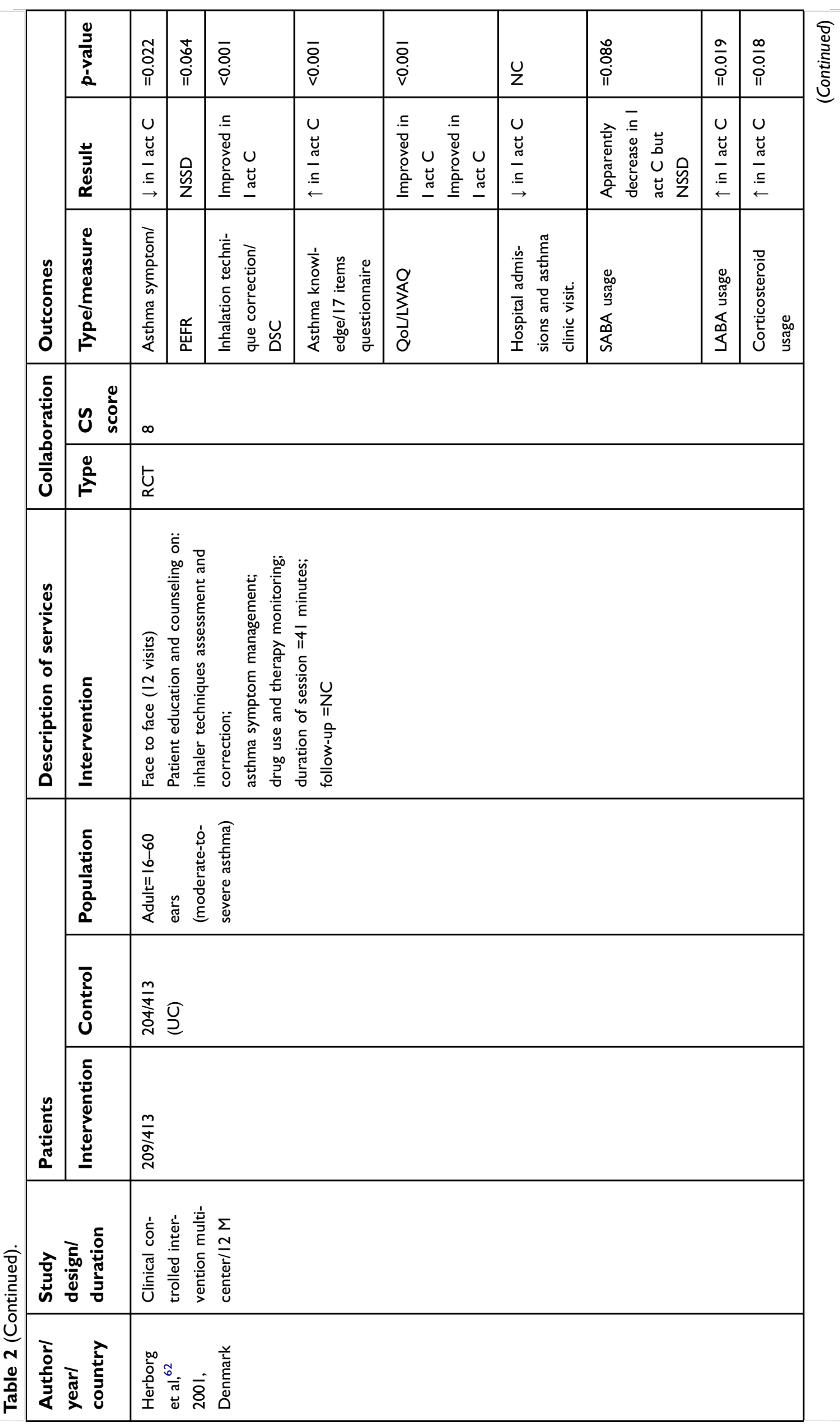




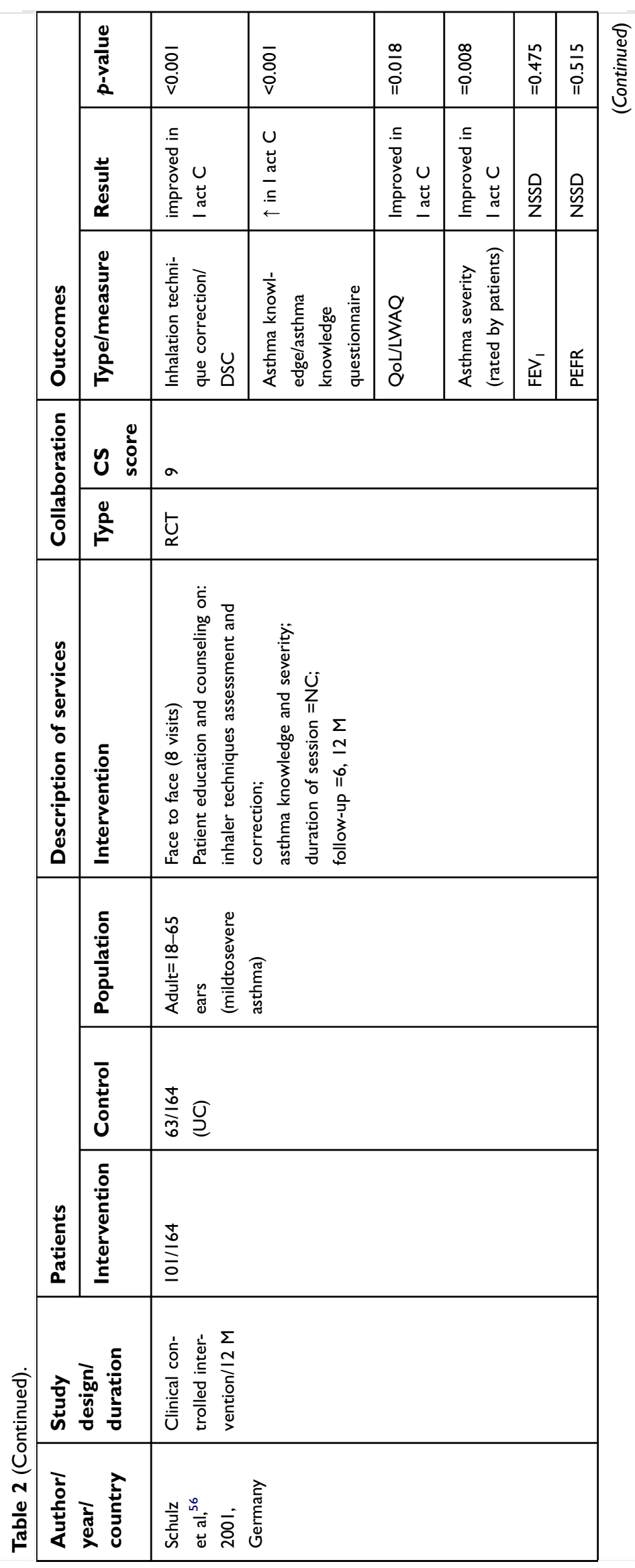




\begin{tabular}{|c|c|c|c|c|c|c|c|c|c|c|c|c|}
\hline \multirow[b]{3}{*}{ 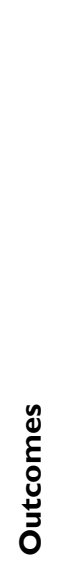 } & $\frac{0}{\frac{0}{\pi}}$ & $\begin{array}{l}\frac{n}{0} \\
\stackrel{0}{0} \\
\mathrm{v}\end{array}$ & $\begin{array}{l}\overline{\dot{0}} \\
\dot{0} \\
\mathrm{v}\end{array}$ & $\begin{array}{l}\infty \\
\stackrel{0}{0} \\
\stackrel{v}{v}\end{array}$ & $\begin{array}{l}\overline{0} \\
\text { i } \\
\text { ॥ }\end{array}$ & $\begin{array}{l}\bar{\delta} \\
\dot{0} \\
\dot{v}\end{array}$ & $\begin{array}{l}\bar{\delta} \\
\dot{0} \\
\dot{v}\end{array}$ & $\begin{array}{l}\overline{8} \\
\dot{0} \\
\mathrm{v}\end{array}$ & $\begin{array}{l}\text { ơ } \\
\text { Oi }\end{array}$ & $\mathrm{U} \mathrm{u}$ & $\begin{array}{l}\overline{8} \\
\dot{0} \\
\dot{v}\end{array}$ & $\begin{array}{l}\infty \\
\stackrel{0}{0} \\
\text { II }\end{array}$ \\
\hline & 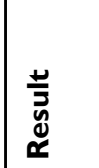 & $\begin{array}{l}U \\
\stackrel{U}{\Xi} \\
\stackrel{\Xi}{\rightarrow} \\
\rightarrow\end{array}$ & $\begin{array}{l}U \\
\stackrel{U}{\sigma} \\
\subseteq \\
\rightarrow\end{array}$ & 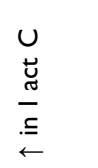 & $\begin{array}{l}U \\
\underset{\Xi}{U} \\
\stackrel{.}{\subseteq} \\
\rightarrow\end{array}$ & $\begin{array}{l}U \\
\stackrel{u}{J} \\
. \subseteq \\
\rightarrow\end{array}$ & 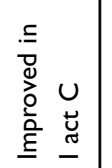 & 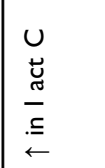 & $\begin{array}{l}U \\
\stackrel{U}{\Xi} \\
\stackrel{.}{\subseteq} \\
\leftarrow\end{array}$ & 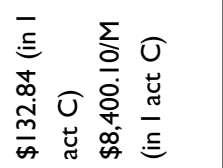 & 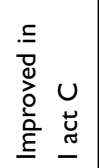 & 莺 \\
\hline & 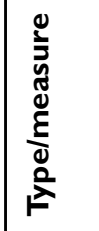 & 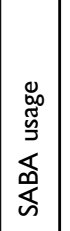 & 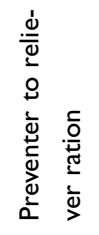 & 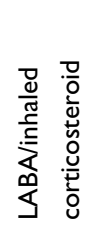 & 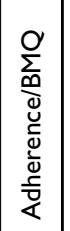 & 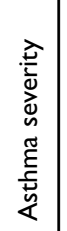 & 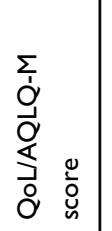 & 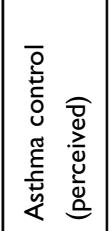 & 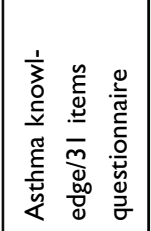 & 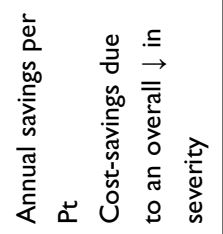 & 岀 & 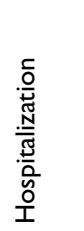 \\
\hline \multirow{2}{*}{ 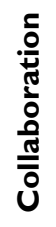 } & บั & in & & & & & & & & & & \\
\hline & 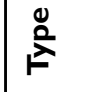 & $\underset{\swarrow}{\longleftarrow}$ & & & & & & & & & & \\
\hline 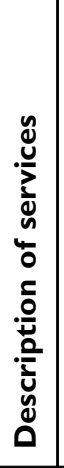 & 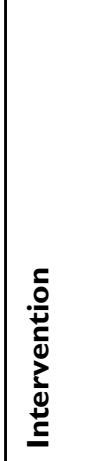 & 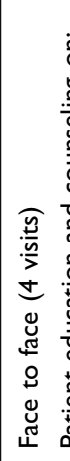 & 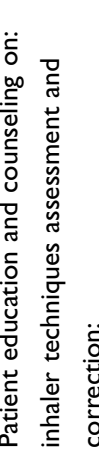 & 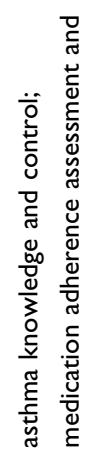 & 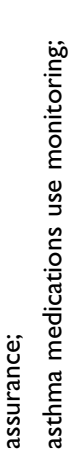 & 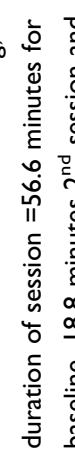 & 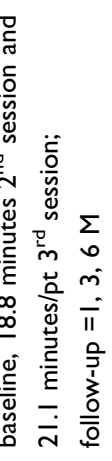 & & & & & \\
\hline \multirow[b]{3}{*}{ 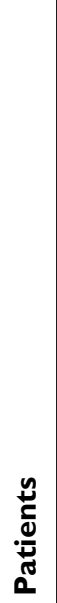 } & $\begin{array}{l}\frac{5}{0} \\
\frac{0}{2} \\
\frac{0}{3} \\
0 \\
0\end{array}$ & 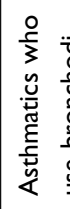 & 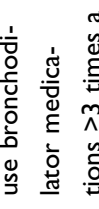 & 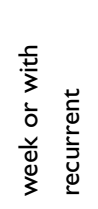 & & & & & & & & \\
\hline & 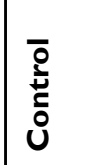 & $\underset{\sim}{\stackrel{o}{o}}$ & 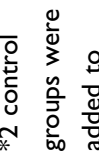 & 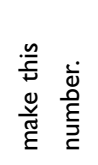 & & & & & & & & \\
\hline & 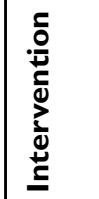 & $\frac{\hat{0}}{\stackrel{m}{m}}$ & & & & & & & & & & \\
\hline \multicolumn{2}{|c|}{ 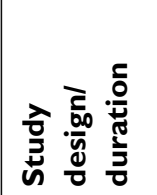 } & 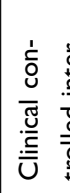 & 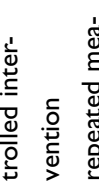 & ڤั & 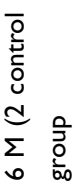 & & & & & & & \\
\hline \multicolumn{2}{|c|}{ 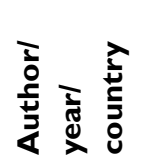 } & 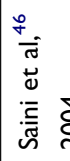 & 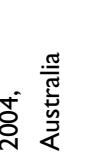 & & & & & & & & & \\
\hline
\end{tabular}




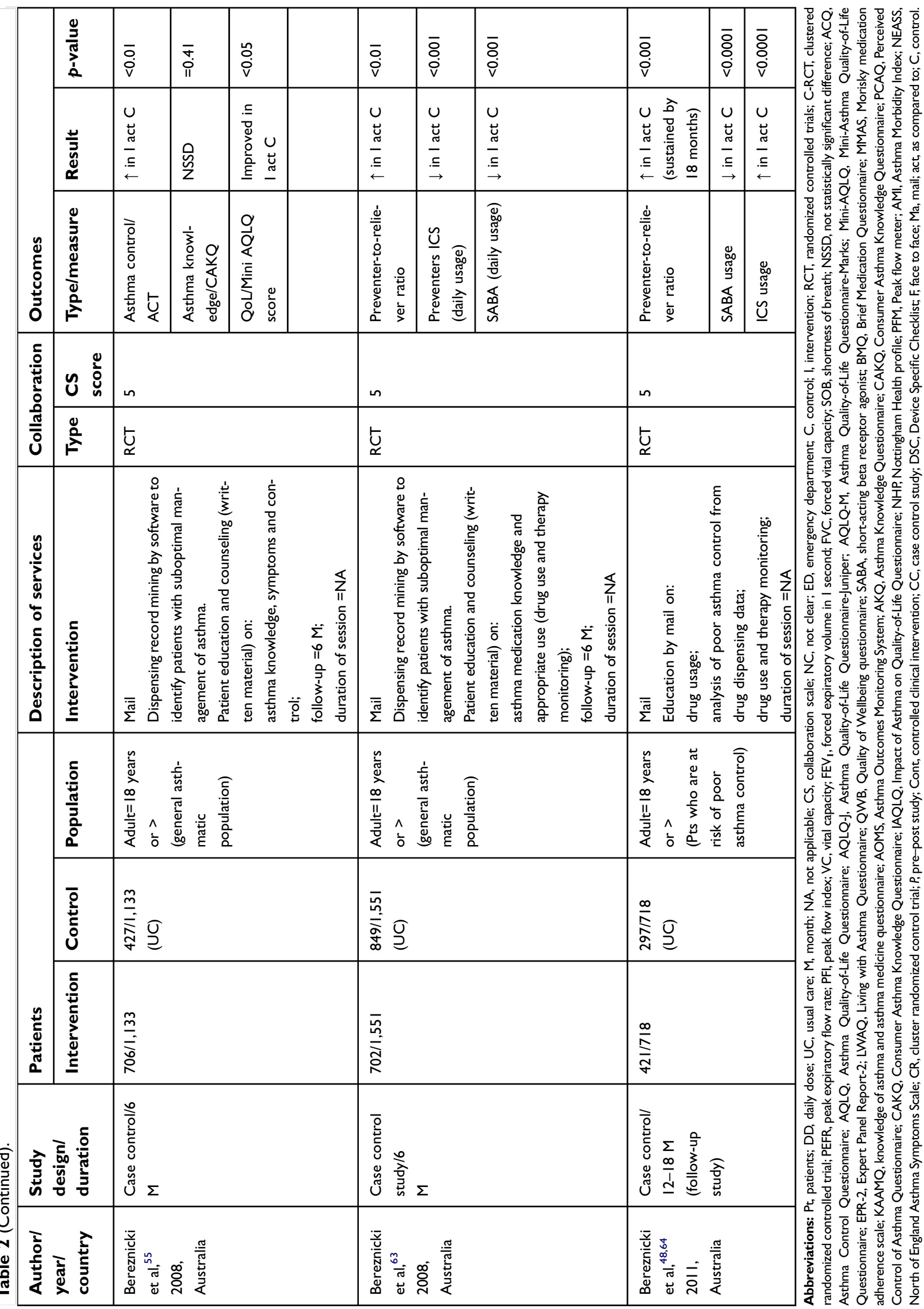




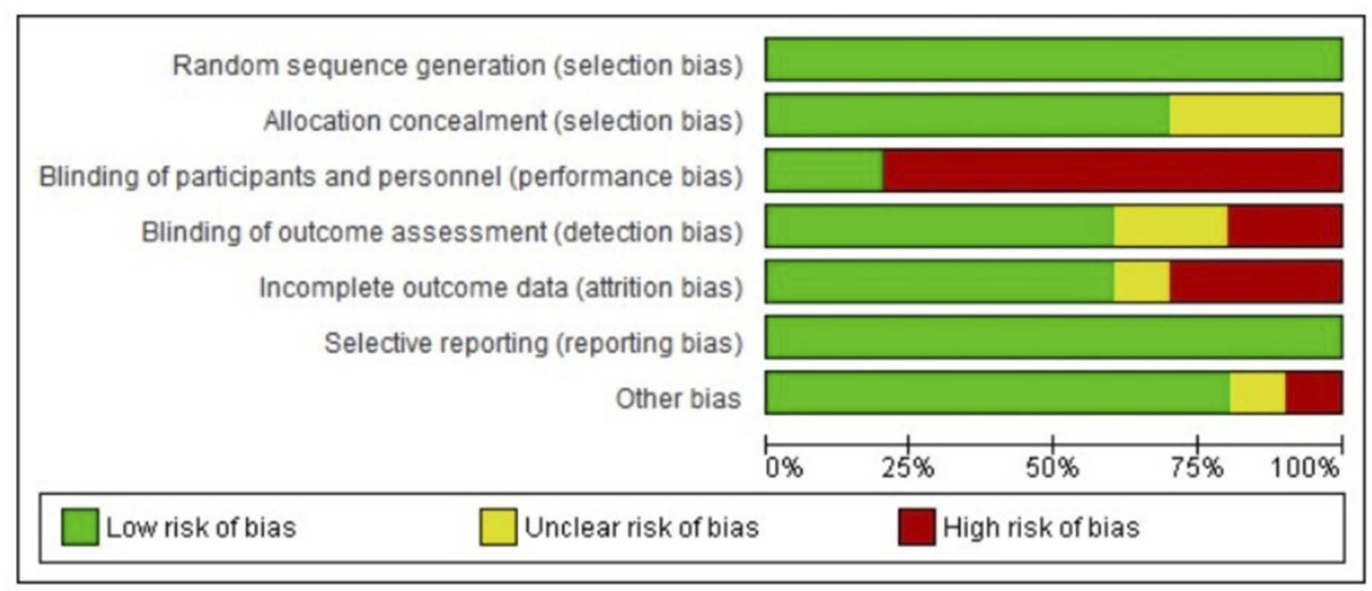

Figure 2 ROB Cochrane graph.

(b) Unfavorable or non-significant (where the study clearly mentions a $p$-value more than 0.05 , indicating no significant improvements by CP's intervention or no difference between intervention or control group).

The summary of the narrative synthesis of the impact of the CP-GP collaborative interventions on all outcomes are summarized in Table 2 and Figure 6.

\section{Clinical outcomes}

Eight studies reported the intervention outcomes on PEFR values from baseline to end-point. Of these, four studies concluded significant differences in improvement in PEFR between the intervention and control groups. However, four studies delineated no significance difference. Similarly, for $\mathrm{FEV}_{1}$, four out of five studies showed no significant difference made by the intervention. $\mathrm{FEV}_{1} / \mathrm{FVC}$ was measured in two studies, both showed non-significant improvements in the intervention as compared to the control group.

Asthma symptoms described as night time or day time wheezing and chest tightness, were evaluated in ten studies. Of these, nine posed significant positive results. Two studies demonstrated mixed results (positive for certain symptoms and not significant for others), and one study concluded no significant change. Nevertheless, the overall impact was positive.

Outcome on asthma severity was reported in five studies. The definition of asthma severity varies across the studies; nevertheless, all studies hold significant impact of interventions.

There were ten studies which explored asthma control as an outcome. Asthma control was defined by a specific score achieved on instruments deployed to measure the asthma control. These instruments were the Asthma Control Questionnaire (ACQ), Asthma Control Test (ACT), and Perceived Control of Asthma Questionnaire (PCAQ), and one other. Two studies showed non-significant results, as compared to eight significant studies.

A total of nine studies reported SABA usage in asthma patients, where seven hold a significant decrease in SABA usage in the intervention group, while one was non-significant, and one did not provide any conclusive data. Similarly, LABA use was discussed in four studies, three significant and one did not provide the data at end. Corticosteroids use was reported in ten studies; out of these, five were significant, four non-significant, and one study did not report the data. Another important parameter in drug usage in asthma patients was preventerto-reliever ratio, which was highlighted in four studies, and these studies demonstrated significant improvement in the intervention group. The impact of GPs-CPs collaboration on asthma drug use. Two studies ${ }^{15,43}$ showed mixed results (improvement in one drug used but no change in another anti-asthmatic drug used).

Eleven studies applied educational intervention to the correct inhalation technique. All studies recorded significant improvement in patients' inhalation techniques.

The effects of these interventions on the number of hospital visits were evaluated in nine studies. Three studies mentioned the lack of data to reach any conclusion, while, the six remaining studies, except one, all reported non-significant results. On the other hand, an emergency room visit was an intended outcome in six studies, three studies could not reach to a conclusion because of limited data to perform statistical operation, while two studies documented non-significant results and one held significance. 


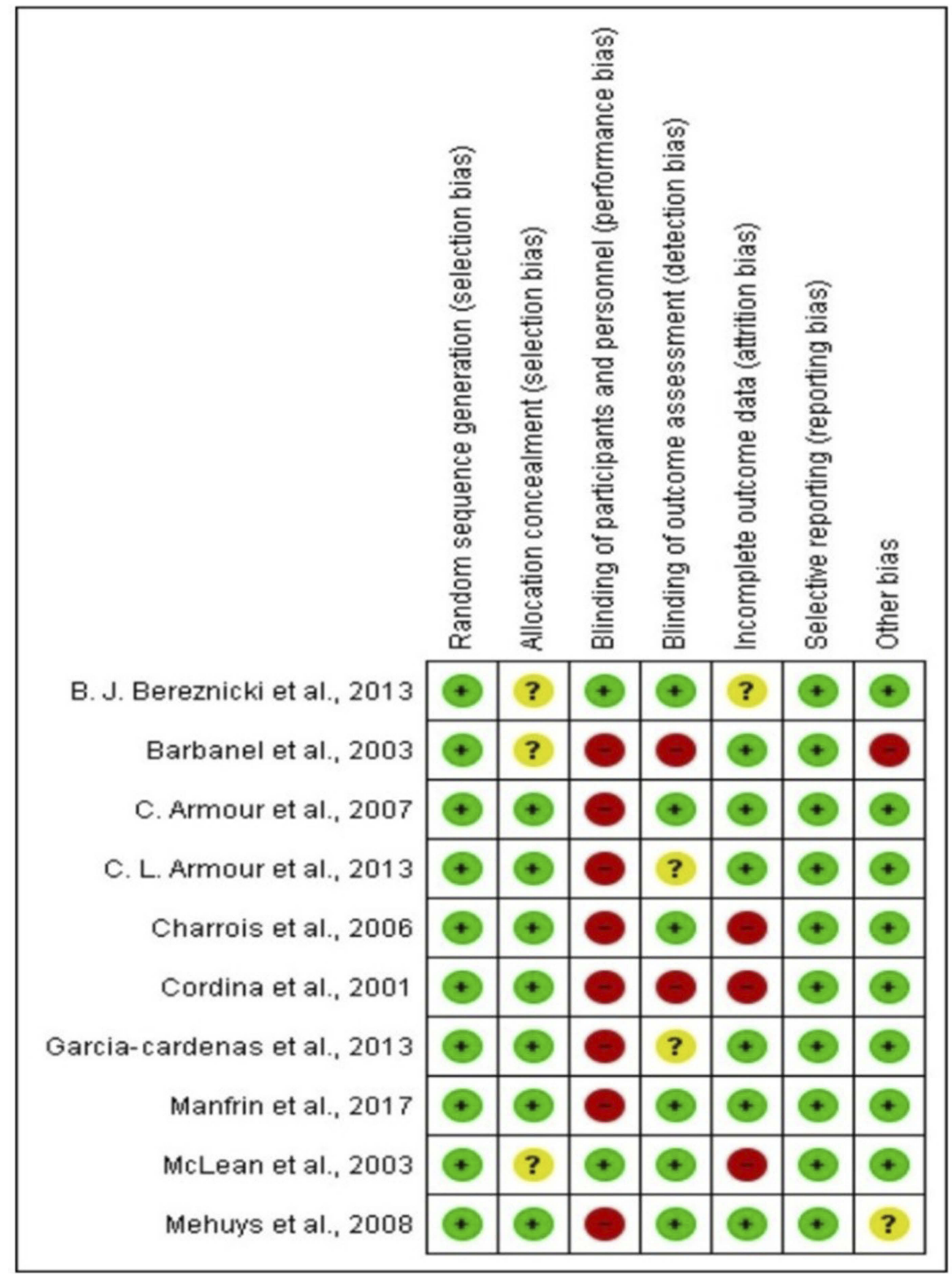

Figure 3 ROB Cochrane summary.

Note:,+- and ? show low, high or unclear risk of bias, respectively.

\section{Humanistic outcomes}

Other reported outcomes of GPs and CPs collaborative practice in asthma management include assessment of medication adherence, asthma drug use, and improvement in inhalation technique and knowledge of asthma. Eight studies reported medication adherence outcomes, where all of them are significant. In those studies, measurement of medication adherence was done using various tools, such as the Morisky medication adherence scale (MMAS) $(n=3)$, Brief Medication Questionnaire (BMQ) $(n=3)$, and the remaining two used self-reported questionnaires.
Patients' knowledge on asthma was assessed in 11 studies. Nine studies showed significant improvement on patients' asthma knowledge and medicine used in the asthma after intervention. This improved knowledge made patients better control and manage asthma. Only two studies reported non-significant changes. There is a considerable variation in the instruments used to measure asthma knowledge reported in those studies. Still, four studies used the Consumer asthma knowledge questionnaire (CAKQ), and three studies deployed the Asthma knowledge questionnaire (AKQ), while all other studies 


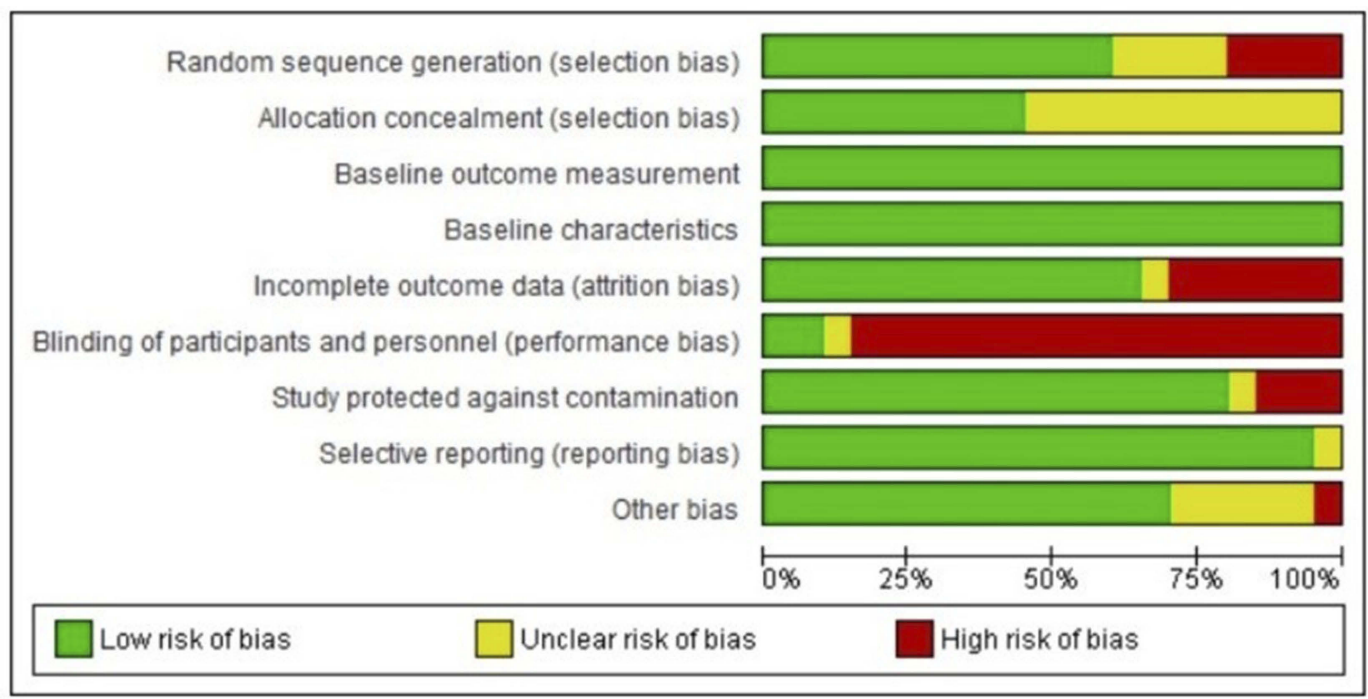

Figure 4 EPOC graph.

used different instruments (21-item questionnaires, Knowledge of asthma, and asthma medicine questionnaire).

Fifteen studies reported the impact of GPs-CPs collaborative practice on patients QoL. Of these, 12 studies reported significant improvement in QoL in the intervention group, while no significant difference between the groups was reported in three studies. Five studies used the Living with Asthma Questionnaire (LWAQ) as a tool to measure QoL, while seven studies administered various forms of the Asthma Quality-of-Life Questionnaire(AQLQ: AQLQ-J, AQLQ-M, AQLQ-mini, AQLQ, IAQLQ), and three studies incorporated others.

\section{Economic outcomes}

Economic outcomes were only reported in five studies. All five studies mentioned value for money of the collaborative practices in various study designs. The most recent national level study in Italy is the most promising one, where cost-effectiveness analysis (the difference in costs from the public healthcare and society perspectives) by the end of study proved the hypothesis that CPs' provided interventions were more cost-effective than usual care, and the probability of being cost-effective increased from $51.50 \%$ at 3 months to $100 \%$ at 9 months. $^{43}$ Another study ${ }^{44}$ cited a cost benefit ratio of CP's interventions as $\$ 5.71$ returned for every $\$ 1.00$ invested as compared to usual care. Similarly, the largest study in the US demonstrated the financial trends in the sense of a direct cost saving of $\$ 1,61,187 / \mathrm{pt}$./Y and an indirect cost saving of
$\$ 1,230 /$ pt./Y of these interventions. ${ }^{45}$ Finally, a study in Australia concluded annual savings per patient Australian $\$ 132.84$, but $p$-values were not mentioned. ${ }^{46}$

\section{Evidence base through meta-analysis}

The step by step procedure and results of all meta-analyses of each outcome are presented in Tables 3 and 4, while larger high definition images of meta-analyses are also provided in Appendix II. Out of 14, 11 outcomes were concluded in favor of CP-GP collaborative interventions with different magnitude of ES. Eight outcomes (Asthma Severity, Asthma Control, Asthma symptoms, PEFR, SABA Usage, Hospital visit, Adherence and QoL (AQLQ, LWAQ) demonstrated a small ES $(d \geq 0.2)$, three outcomes (Inhalation Technique, ED visit, and Asthma Knowledge) witnessed medium ES ( $d \geq 0.5)$. Inhalation technique yielded large ES $(\mathrm{d} \geq 0.8)$ in RCTs subgroup analysis. However, three outcomes (FEV, Corticosteroids usage, and Preventer to Reliever ratio) did not hold significant ES $(\mathrm{d}<0.2)$ and, thus, remain inconclusive. The collaboration was shown to be value for money in the economic studies in narrative synthesis; however, a limited number of studies did not allow pooling of data in metaanalysis. For all outcomes, the conclusion based on effect size is summarized in Table 5.

\section{Discussion}

To our knowledge, this is the first review and meta-analysis of CP-GP collaborative intervention in asthma management. The evidence base from this systematic review witnessed the increasing level of collaboration between CPs and GPs, and 


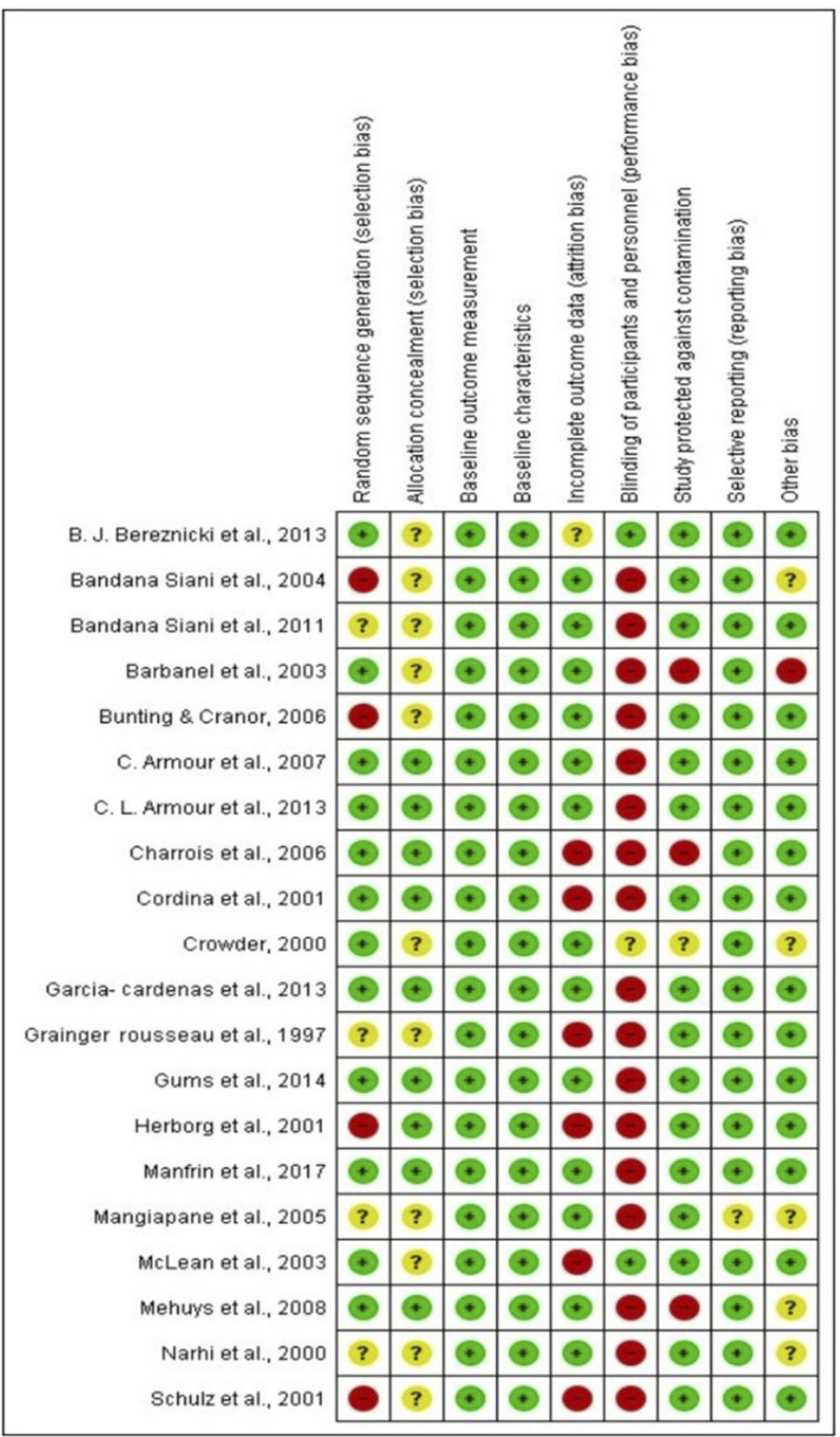

Figure 5 EPOC summary.

Note: +, - and ? show low, high or unclear risk of bias, respectively.

confirmed the clinical, humanistic, and economical superiority of the collaborative interventions to improve asthma outcomes as compared to usual care. This review distinguished many studies reporting the collaborative interventions. Involving CPs in clinical decision-making in collaboration with GPs is an emerging trend worldwide, and a significant number of studies were conducted on collaborative practice between $\mathrm{CP}$ and GP in recent years in European countries. ${ }^{5,43,47,48}$

The results were consistently significant for improvements in inhalation technique, which isa core element of better asthma control. Similarly, the need of rescue medicine decreased (high usage of rescue medication means poor 


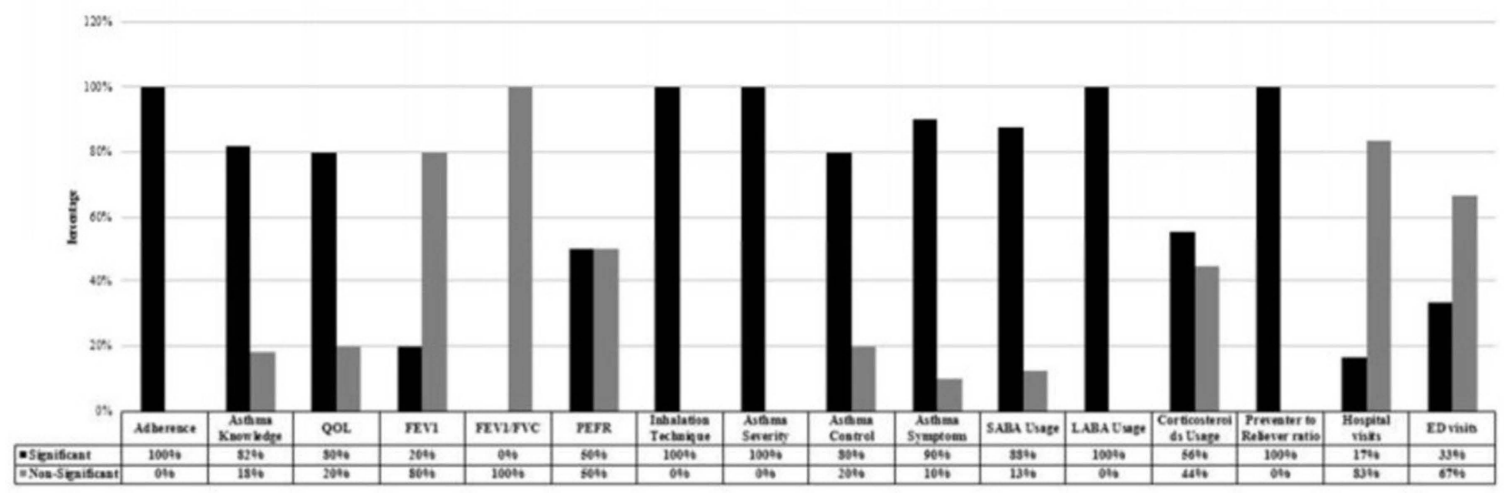

Figure 6 Narrative synthesis of the included studies.

Abbreviations: ED, emergency department; FEV, forced expiratory volume; FVC, forced vital capacity; LABA, long acting beta-agonist; PEFR, pulmonary expiratory flow rate; QoL, quality-of-life; SABA, short acting beta-agonist.

asthma control) which contextually proved the better management of asthma in the intervention group.

Achieving and sustaining asthma control and improvement in QoL are the foundation of asthma management. These interventions on the whole have significantly improved not only the medication use and management, but also substantially contributed towards better control and self-management, as well as enhanced knowledge and QoL. The possible reasons for this improvement could be attributed to:

(a) Patient's enhanced knowledge of diseases and medication which may halt the patient's false belief on pseudo medicine or therapies.

(b) Patient's better understanding of the identification of triggers and ways to minimize or handle effectively.

(c) Written self-management strategies.

(d) Corrected inhalation technique.

(e) Better understanding and adherence to medication regimen (the appropriate use of reliever and preventive medication).

(f) Refined collaboration of caregivers, where they update and refer patients to each other for medications review, advice, education, and counseling, projecting a sense of ownership of patients.

The current review presents a different but important consideration; compared with other systematic reviews which cover individual CPs interventions, the focus was on collaborative practice between $\mathrm{CP}$ and GP. An interesting observation made in this review was the presence of a general correlation between the impact of intervention on patients' multiple outcomes and score on collaboration scale; that is, the higher the collaboration-score, the higher would be the positive patients' outcomes. For instance, all studies with collaboration-scores 10/10 (indicator of highest form collaboration) has significantly improved all outcomes (clinical, humanistic, and economical) in the intervention group. ${ }^{15,43,45,47,49,50}$ On the contrary, the studies with a Collaboration scale score $\leq 5$ have shown multiple outcomes with no significant statistical difference in the intervention group as compared to the control group. ${ }^{42,51-53}$

In his doctoral thesis, Amirthalingam ${ }^{54}$ pointed out that collaboration between CP-GP rather than solo CPs' interventions could produce more promising results in clinical and humanistic outcomes in chronic diseases like hypertension. Our review seconded the findings for asthma and established the fact that collaborative intervention in asthma management is promising, especially for clinical and humanistic outcomes. The positive impact of collaborative practice on asthma management has implications for practice and policy. The policy-makers should consider the untapped potential and formulate policies which favor the role of CPs in medication management, especially in chronic diseases. Positive experiences from this collaborative model approach of chronic diseases management should be taken as a lesson for countries where the concept of single care provider is still widely operated.

A notable point which may constitute high heterogeneity in the results was the use of diverse data collection tools (questionnaires) in the added studies for the same outcome. For instance, QoL, asthma knowledge, and adherence to medications were assessed in various studies using three, four, and two different types of questionnaire, respectively. This made the situation overly complicated, 


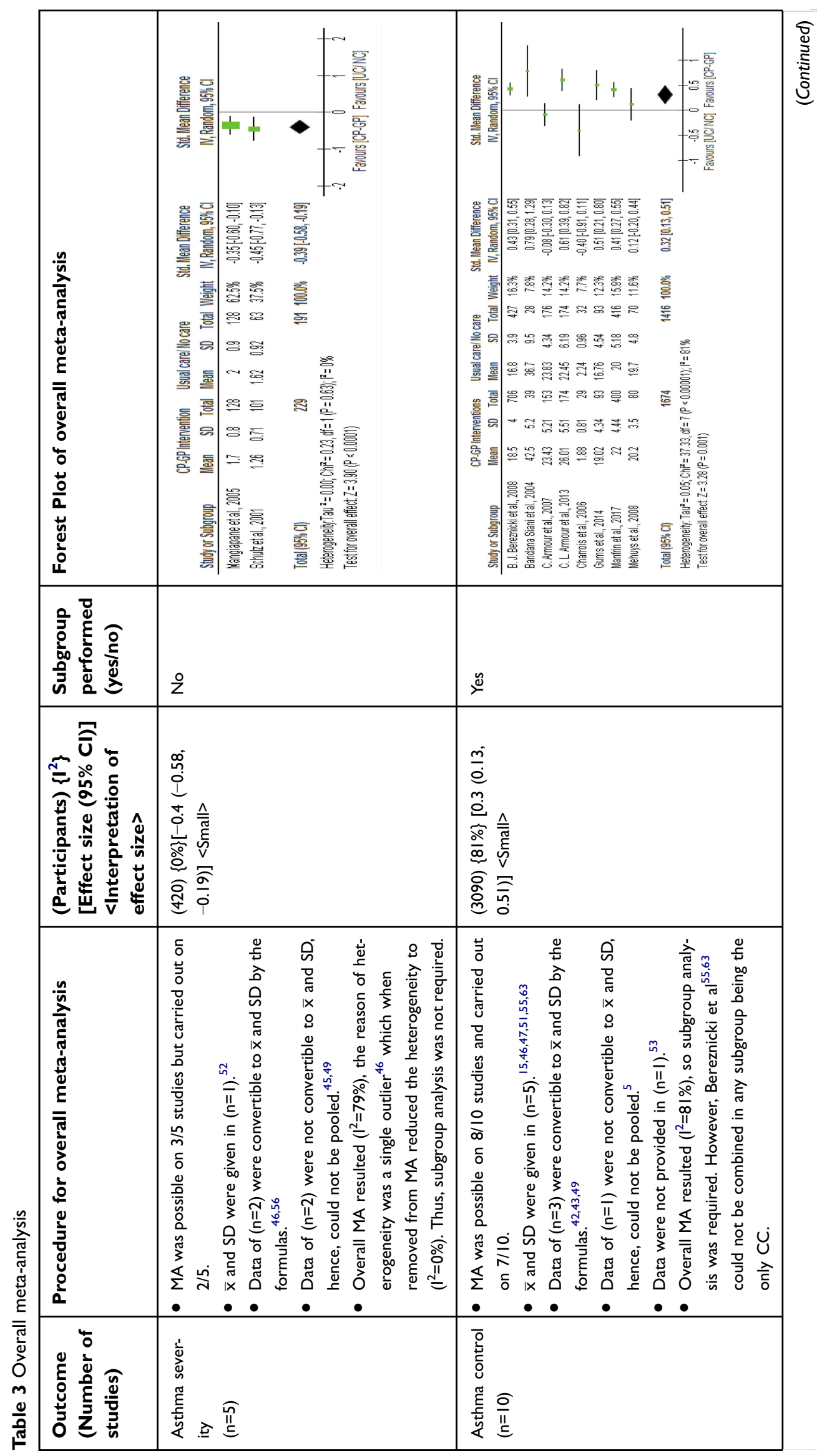




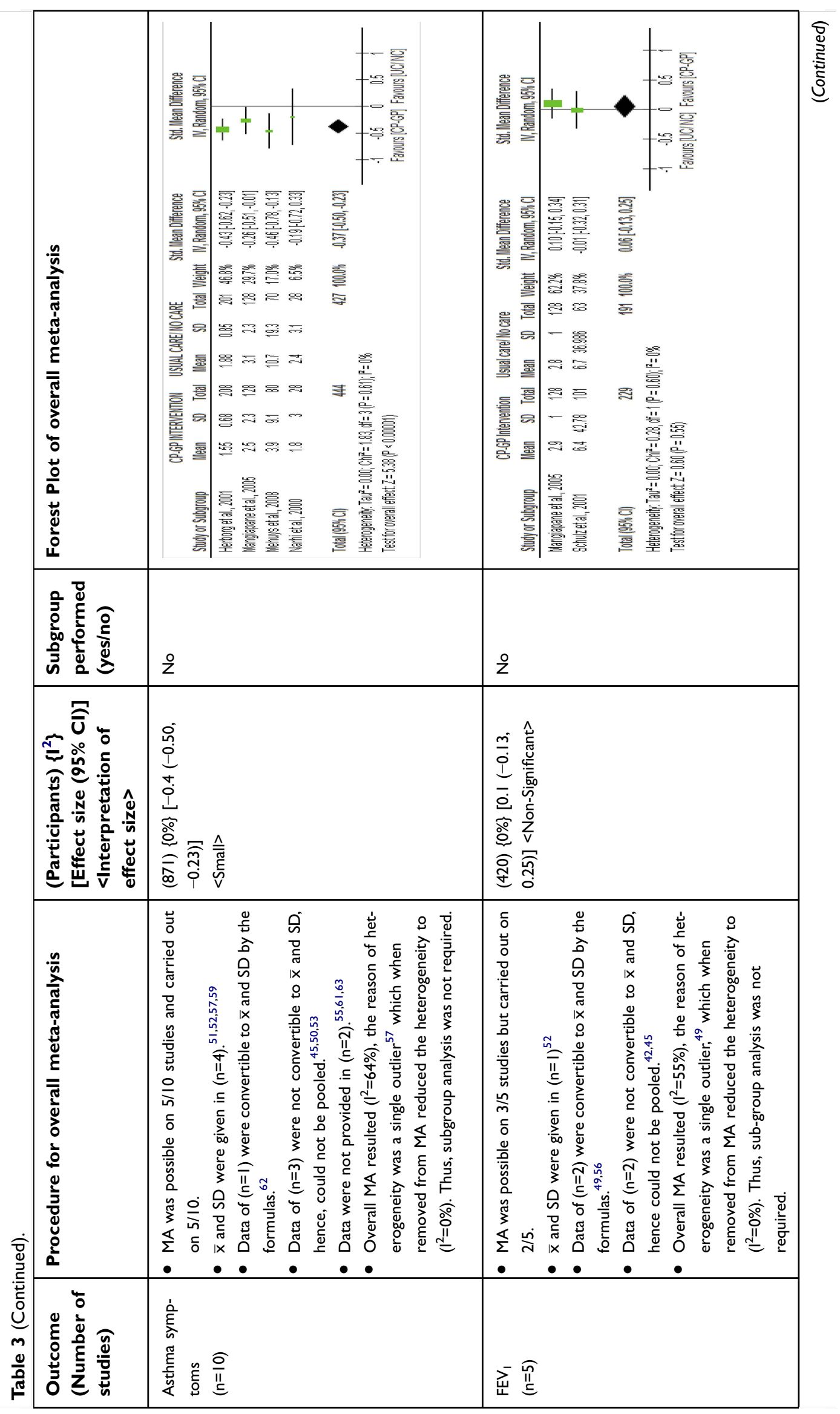




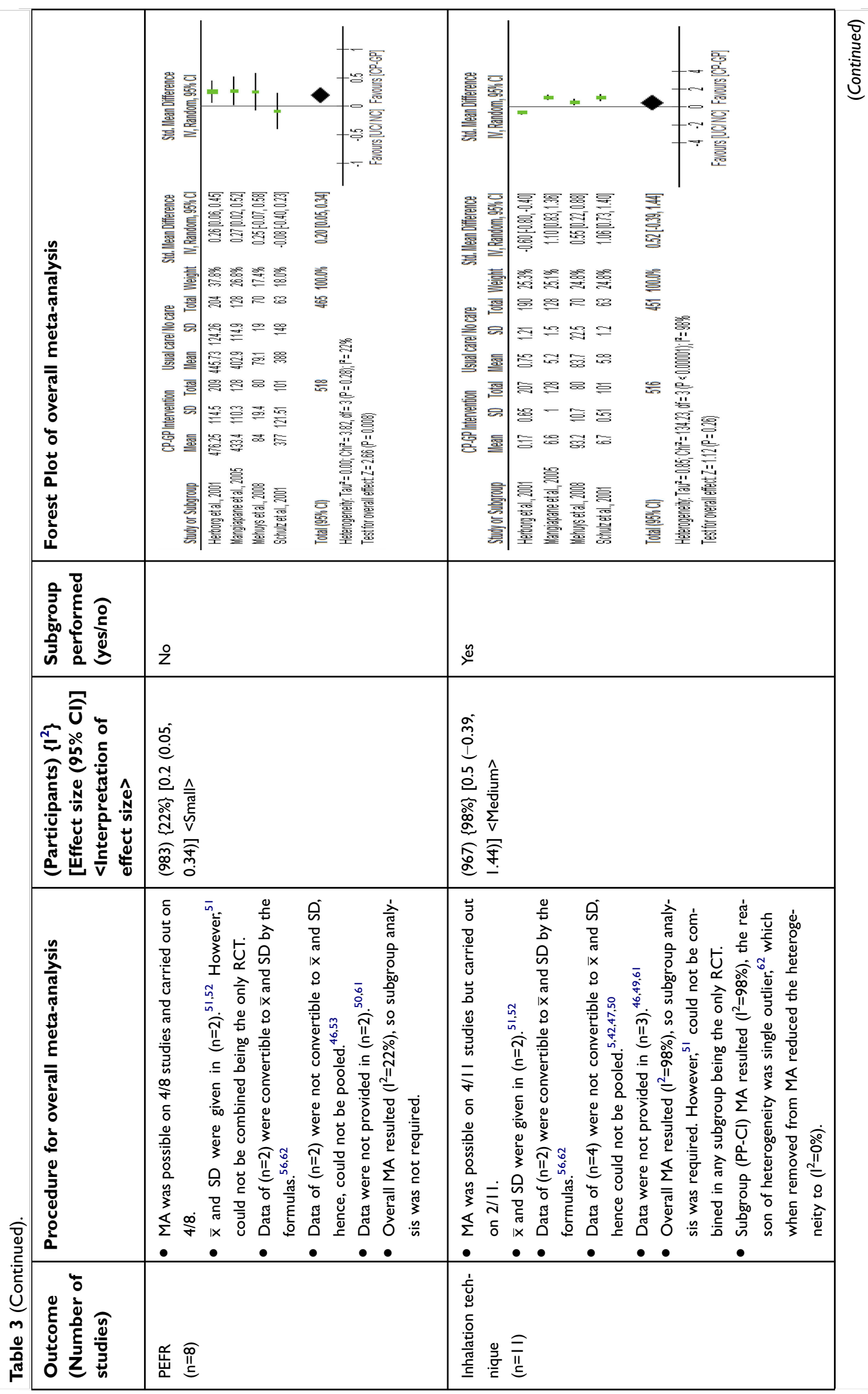




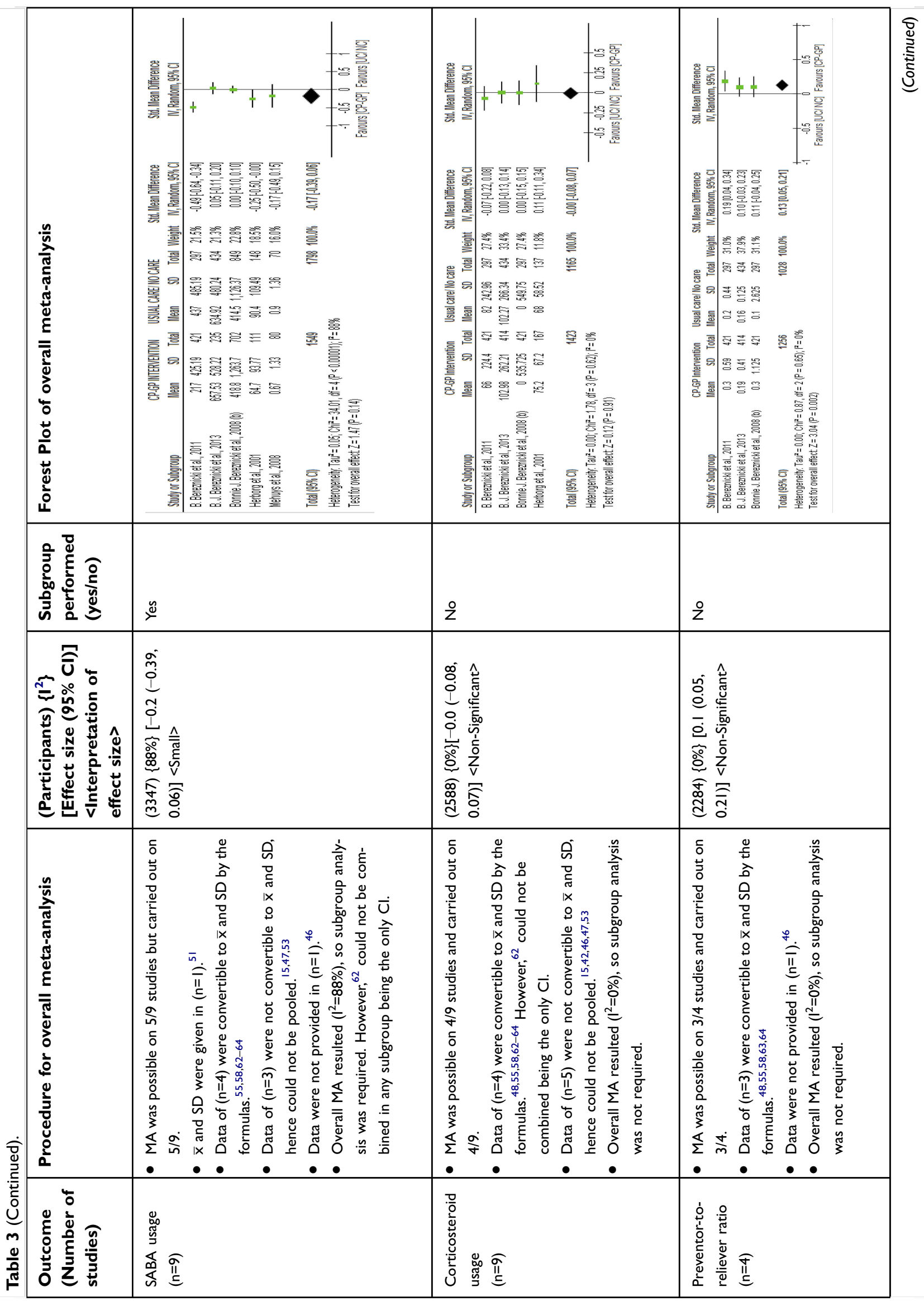




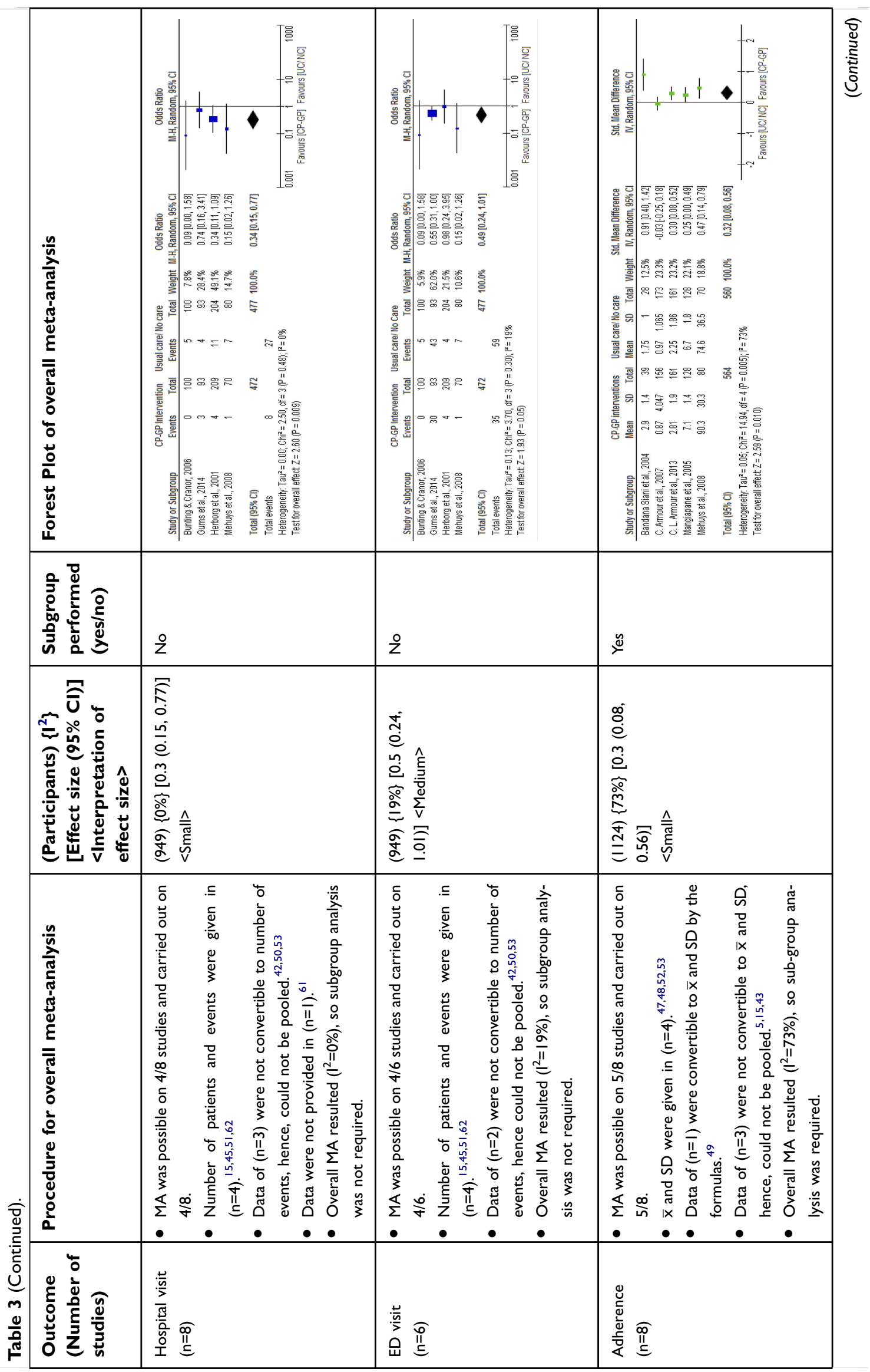




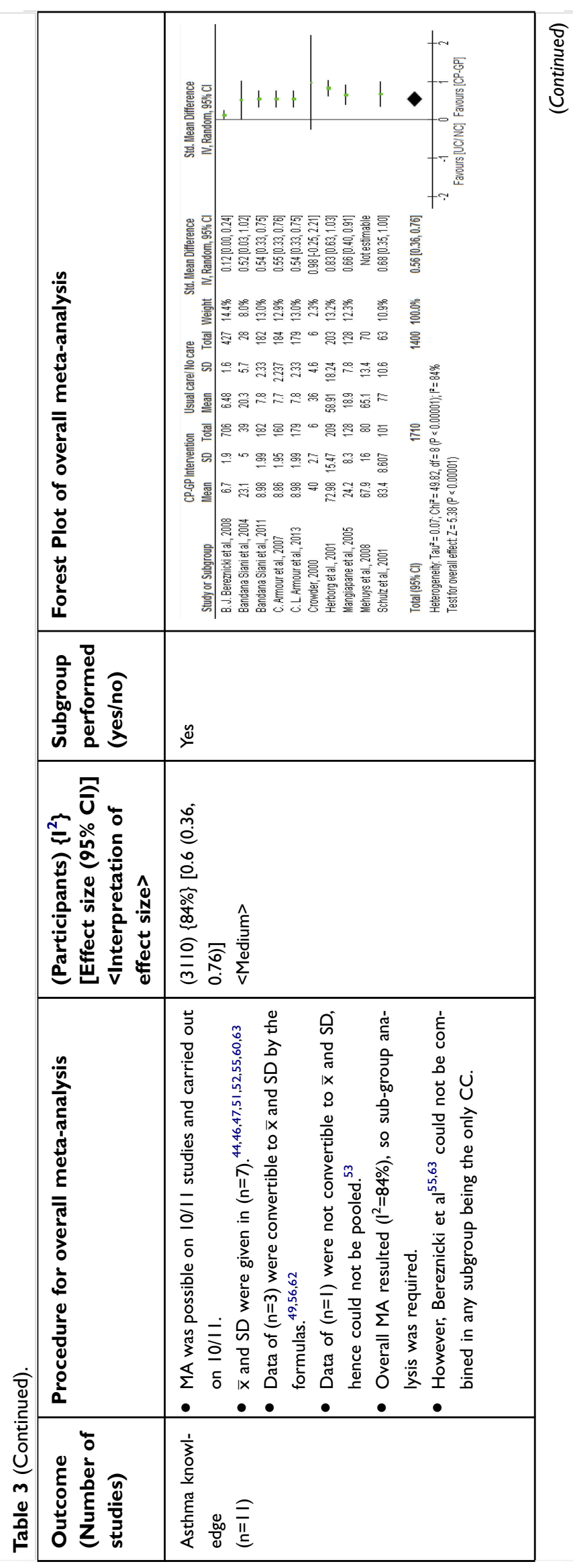




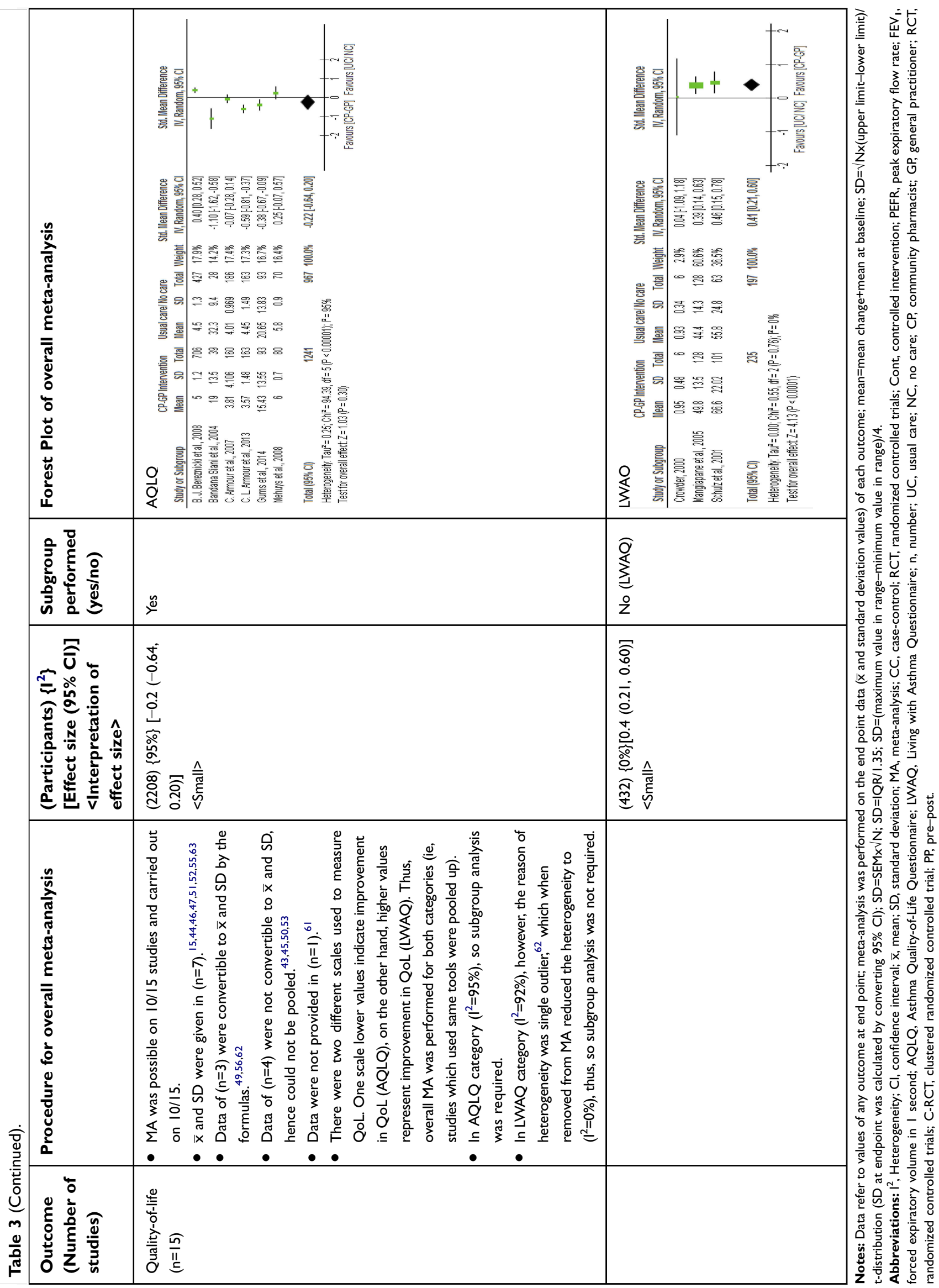




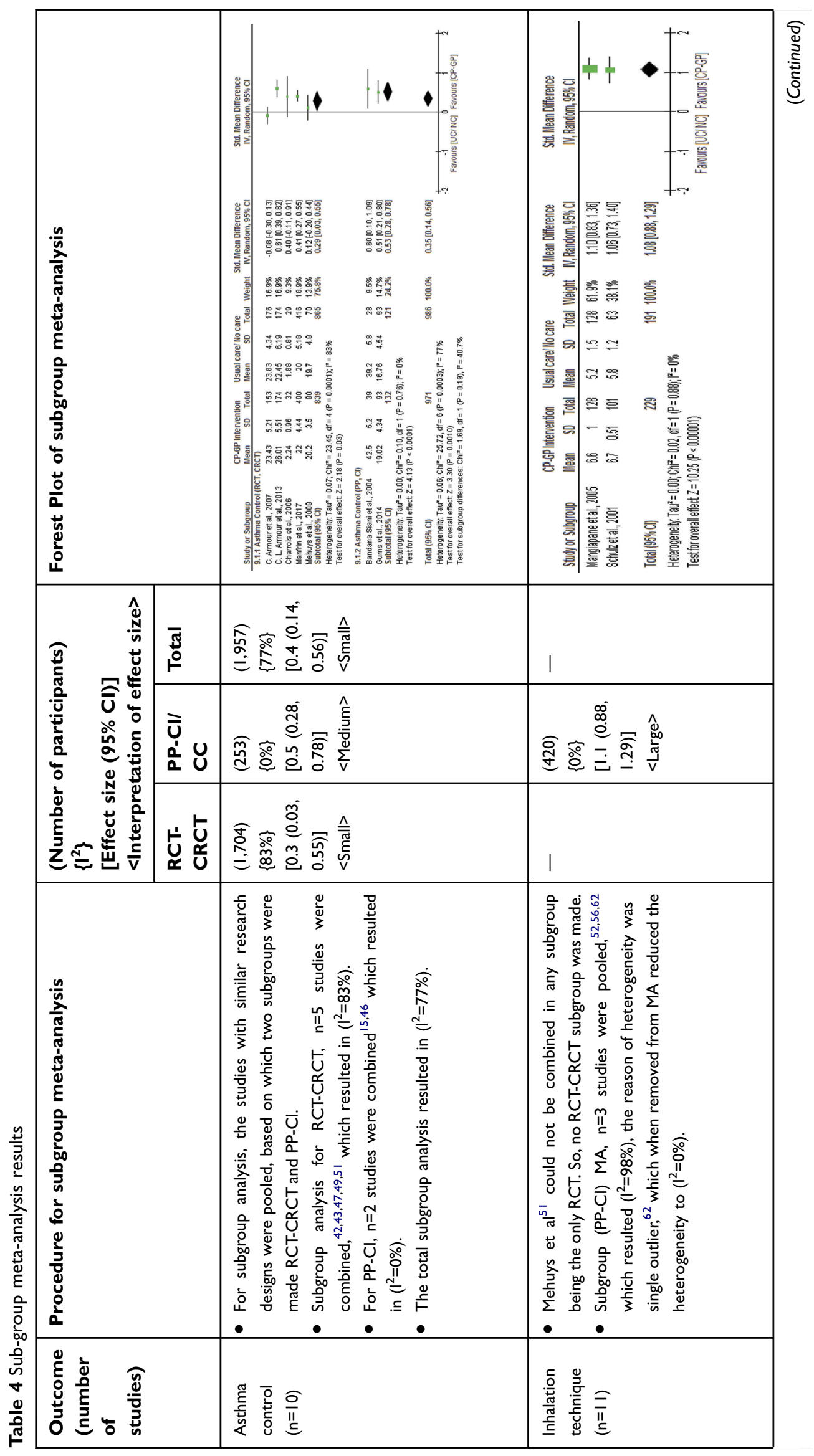




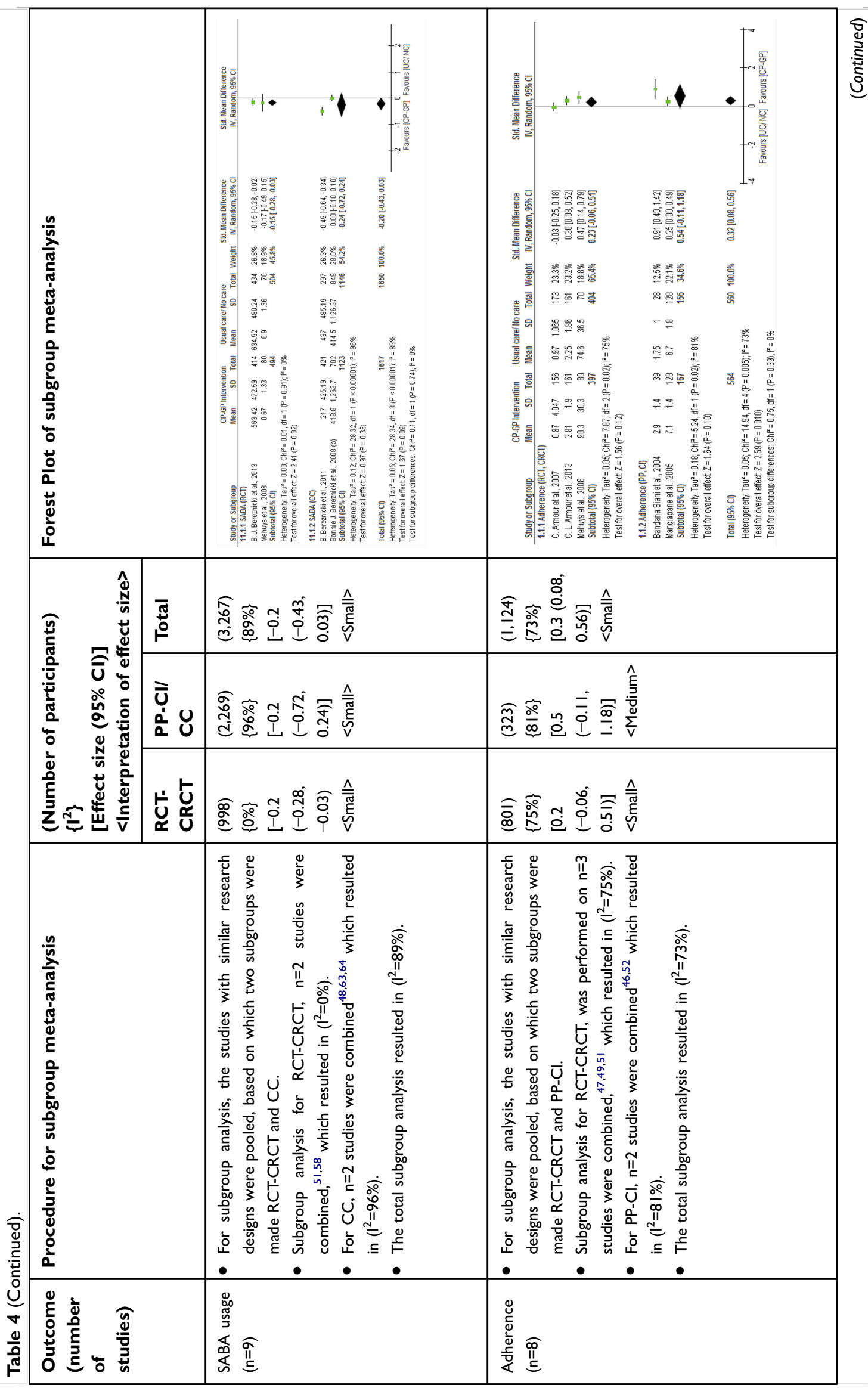




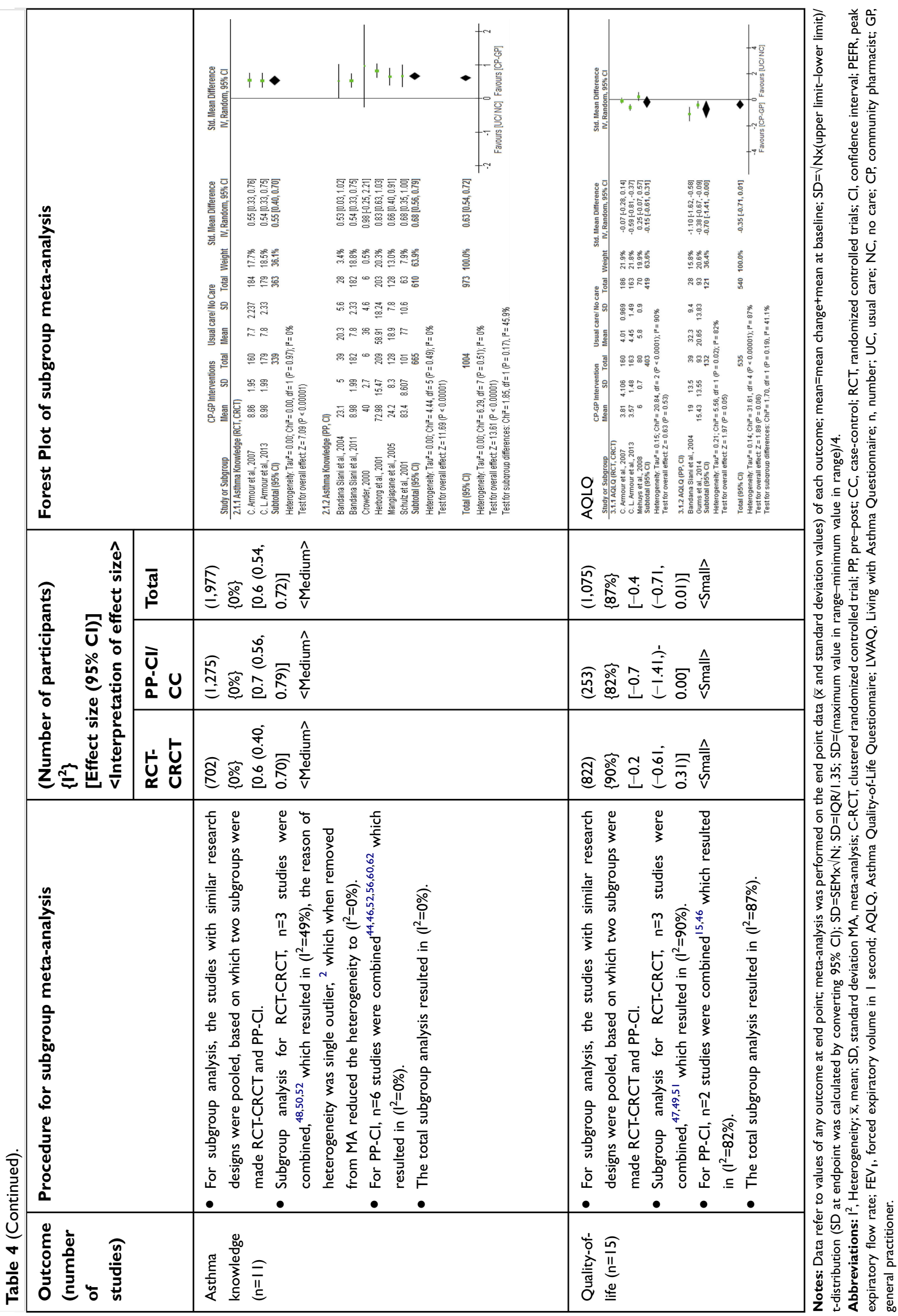




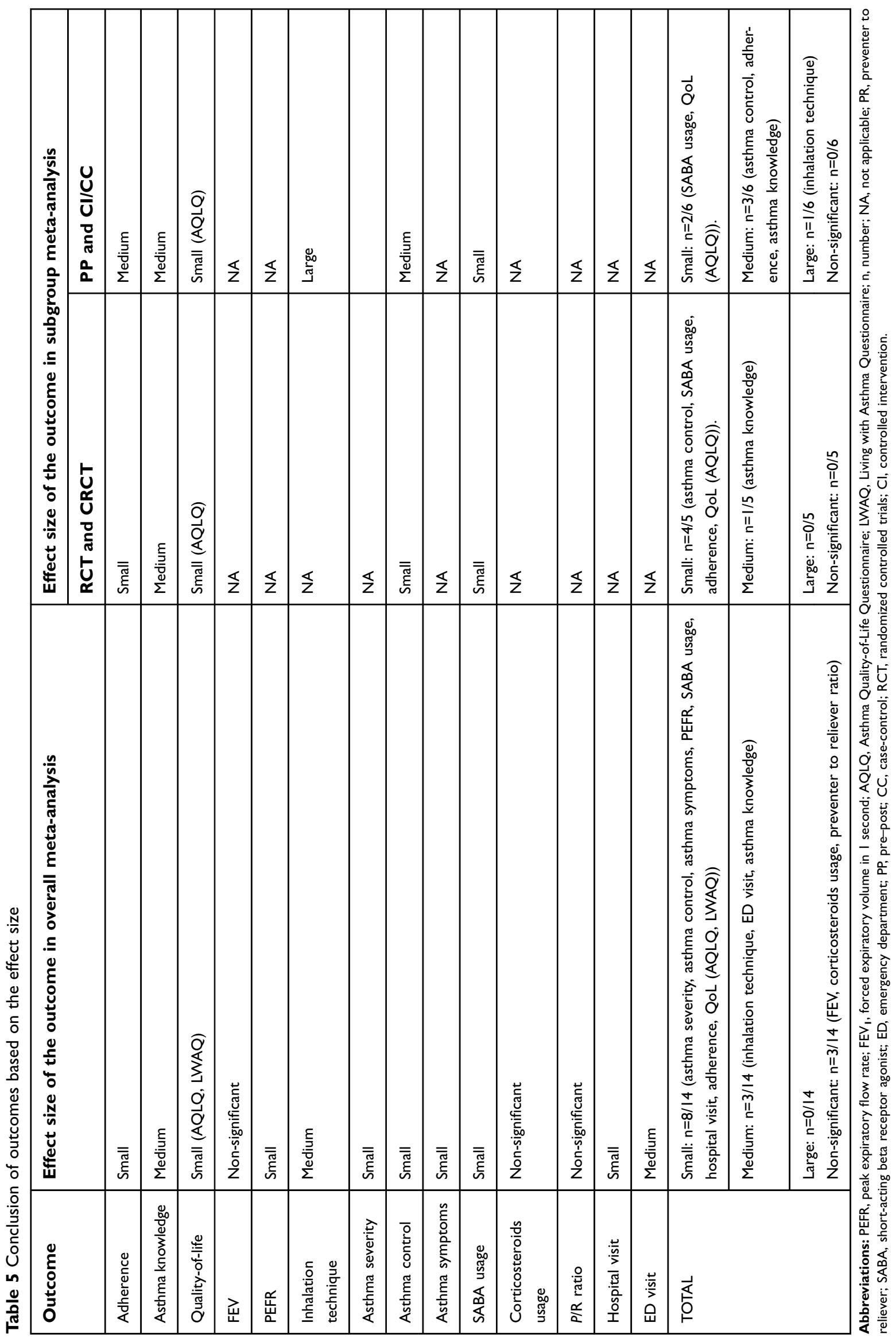


and it needs a standardized approach in future. We gave due importance to heterogeneity and executed subgroup analysis to find out the outliers and reasons of extreme heterogeneity.

In meta-analysis, out of the 11 outcomes which favored collaborative practice, eight outcomes resulted in small and three medium effect sizes. An important point regarding the magnitude of effect size obtained in the metaanalysis of various outcomes needs to be understood clearly. It is irrational to consider a large effect as the only important effect. It must be clear while drawing some conclusions that, for a policy-maker, a small effect size of 0.2 may also hold much attention. It is not just the numerical superiority of ES which matters, it is also the clinical relevance of the magnitude of an outcome, which holds much weight when making a policy change, ie, how big the problem is, and how much healthcare resource it utilizes. For a chronic disease with a huge burden, even a small ES may be of importance for the policy-makers. ${ }^{40,41}$

A recent systematic review and meta-analysis was carried out to pool the data for adherence. ${ }^{24}$ Authors reported significant improvements in adherence. However, the methodology used to conduct meta-analysis was different, and the scope of the review was not focused on collaborative interventions, yet the results hold a similar level of significance, as described in our results, and our data further confirm the strength of the evidence.

The expanded role of CPs in patient care and as a potential resource of relationship building between patient and primary caregiver was evident throughout the studies involved. The active participation of CPs and resultant positive outcomes could be attributed to various factors, which may include but are not limited to a) the advance certification programs on medicine management or pharmacotherapy or diseases management offered by the pharmacist associations in the countries reported (eg, the Australian Association of Consultant Pharmacy or American Pharmacist Association), b) the advance level trainings and modern curricula of pharmacy education with more emphasis on pharmacotherapy and patient care for a specific chronic disease management (asthma, diabetes, hypertension), and last but never least, c) the emerging scope of financial incentives (reimbursements) for the CPs.

Finally, there were a few economic studies with poor design. It was not possible to pool the studies because of limited data. There is a growing need for well-designed economic studies which appraise CP-GP collaborative practice which may clear the blurred economic picture. This will further facilitate the decision-making in healthcare systems.

The strength of the review is the systematic way evidence is collected, appraised, and depicted in the form of meta-analysis for 14 different kinds of outcomes, where many of the outcomes were not previously quantified. Furthermore, the meta-analysis procedure was discussed in detail, and subgroup analysis was carried out to get a clear picture of effect size.

\section{Limitations}

The following limitation of this review is noted: Every effort was made to make the search extensive, still, because of a lack of accessibility, we could not search International pharmaceutical abstracts and Embase. However, various other databases were added to compensate for this.

It was still difficult to group the outcomes in individual studies to pool the results in a meta-analysis. There were a number of factors which governed these limitations, for instance the medley of methodological designs, differences in patients' population, and prognostic variables, as well as a lack of definite statistical power to reach a conclusion. Besides, for continuous outcomes (ACT, ACQ scores, or asthma severity etc.), many studies have serious flaws which halt their addition into meta-analysis; for instance, missing standard deviation of mean change for control group, ${ }^{5,55}$ lack of control group measures, ${ }^{47,52}$ and lack of standard deviation of mean for both groups. ${ }^{46,56}$ We tried to convert the available data to the format which is suitable to add in the meta-analysis, but it was not plausible for all studies. However, for dichotomous outcome (eg, asthma control) a few studies were appropriate for meta-analysis. ${ }^{5,47,51}$ The authors believe the major reason of heterogeneity and a few inconsistent results are primarily linked with the different instrument used to assess the same outcome, as mentioned in the summary table. Apparently, the interventions are similar, but, on a zoomed scale, we find the difference in contents of training, modes of delivery, and duration and frequency of sessions are quite different. Furthermore, the follow-ups were by telephone, ${ }^{57}$ while others use mail ${ }^{48,58}$ or face to face sessions. All these could be taken as a potential source of variability. Besides this, many studies proffer some flaws; for instance, two studies have too few patients in their sample, which make the results a bit dubious and less generalizable. ${ }^{44,57}$ The longest study in the US evaluating the impact of CPs interventions, though, mentioned significant beneficial effects for the patients and 
healthcare system; however, this can be criticized for the free medications which were given to patients as an incentive to attract. $^{45}$ This potential factor can lead to an interpretation that free access to medicine may itself be self-sufficient to have a good impact on patient clinical outcomes, irrespective of the specific intervention. Another considerable drawback in the same study was the CPs interventions cover $75 \%$ of patients, and still $25 \%$ of patients were given the intervention in the hospital, while there was no clear-cut data depicting this separation and, thus, contaminating the study. Furthermore, a pediatric population was also added to the study, but no data accounts for this population. ${ }^{45}$ Finally, the use of different scales in various studies offers a significant limitation, which may lead to non-reproducible results. Hence, there is a need to standardize the methodology, research designs, tools, as well as the intervention. This will further minimize the biases and strengthen the base. Although 11/14 outcomes have shown promising results, we still need further data in the form of large RCTs with standardized tools of research to expand our understanding on this issue.

Despite some inherent limitations in studies included in this review; nevertheless, results were coherent and sufficiently comprehensive for the positive impact of the CP-GP collaboration in inhalation technique correction, medication use, asthma control, knowledge, and adherence. Other outcomes also appear promising, but need well designed clinical trials.

\section{Conclusion}

The community pharmacy setting has emerged as a beneficial point of contact for management of asthma. The overall evidence base is consistent in favor of CP-GP collaborative practice in asthma management and supports implementation of this collaborative practice between CP-GP. The results of this research advocate the active engagement of CPs in asthma management and rational drug use. The evidence is sufficiently comprehensive to favor CPs intervention for inhalation technique correction, asthma knowledge and control, drug adherence, QoL, and rationalizing drug use. The time has reached CPs to individualize patient care plans and practice evidence based pharmaceutical care. The Australian concept of "asthma friendly pharmacies" could serve as one of the millennium goals to make the retail pharmacy setup into a modern future "health hub".

\section{Disclosure}

The abstract of this paper was presented at the Annual Congress on Medicine, as a poster presentation/conference talk with interim findings. The poster's abstract was published in Biology and Medicine, ISSN: 0974-8369. https://www. omicsonline.org/proceedings/a-systematic-review-and-metaanalysis-of-the-impact-of-collaborative-practice-between-community-pharmacist-and-general-practitioner-99343.html. The authors report no conflicts of interest in this work.

\section{References}

1. Global Asthma Network. The Global Asthma Report 2018. The Global Asthma Network: Vol. 5. Auckland, New Zealand; 2018. ISBN: 978-0-473-29125-9 $\backslash \mathrm{r978-0-473-29126-6} \mathrm{(Electronic).}$

2. Global Initiative for Asthma (GINA). Global Strategy for Asthma Management and Prevention. The Global Initiative for Asthma: Fontana, WI; 2018.

3. Burney P, Jarvis D, Perez-Padilla R. The global burden of chronic respiratory disease in adults. Int J Tuberc Lung Dis. 2015;19(1):1020. doi: $10.5588 /$ ijtld. 14.0446

4. WHO. Global Surveillance, Prevention and Control of Chronic Respiratory Diseases. World Health Organization: Geneva; 2007. ISBN 9789241563468 .

5. Garcia-Cardenas V, Sabater-Hernandez D, Kenny P, MartinezMartinez F, Faus MJ, Benrimoj SI. Effect of a pharmacist intervention on asthma control. A cluster randomised trial. Respir Med. 2013;107(9):1346-1355. doi:10.1016/j.rmed.2013.05.014

6. Bradley CL, Luder HR, Beck AF, et al. Pediatric asthma medication therapy management through community pharmacy and primary care collaboration. J Am Pharm Assoc. 2016;56(4):455-460. doi:10.1016/ j.japh.2016.03.007

7. Barton C, Proudfoot J, Amoroso C, et al. Management of asthma in Australian general practice: care is still not in line with clinical practice guidelines. Prim Care Respir J. 2008;18(2):100-105. doi:10.3132/pcrj.2008.00059

8. Wiener-Ogilvie S, Pinnock H, Huby G, Sheikh A, Partridge MR, Gillies J. Do practices comply with key recommendations of the British asthma guideline? If not, why not? Prim Care Respir J. 2007;16(6):369-377. doi:10.3132/pcrj.2007.00074

9. Watkins K, Bourdin A, Trevenen M, et al. Opportunities to develop the professional role of community pharmacists in the care of patients with asthma : a cross-sectional study. NPJ Primary Care Respir Med. 2016;26. doi:10.1038/npjpcrm.2016.82

10. Benavides S, Rodriguez JC, Maniscalco-Feichtl M. Pharmacist involvement in improving asthma outcomes in various healthcare settings: 1997 to present. Ann Pharmacother. 2009;43(1):85-97. doi:10.1345/ aph.1K612

11. Felix J, Ferreira D, Afonso-Silva M, et al. Social and economic value of portuguese community pharmacies in health care. BMC Health Serv Res. 2017;17:1. doi:10.1186/s12913-017-2525-4

12. Alotaibi HS, Shivanandappa TB, Nagarethinam S. Contribution of community pharmacists in educating the asthma patients. Saudi Pharm J. 2016;24(6):685-688. doi:10.1016/j.jsps.2015.06.002

13. Carter BL. Primary care physician-pharmacist collaborative care model: strategies for implementation. Pharmacotherapy. 2016;36 (4):363-373. doi:10.1002/phar.1732

14. Hisashige A. The effectiveness and efficiency of disease management programs for patients with chronic diseases. Glob J Health Sci. 2013;5(2):27-48. doi:10.5539/gjhs.v5n2p27

15. Gums TH, Carter BL, Milavetz G, et al. Physician-pharmacist collaborative management of asthma in primary care. Pharmacotherapy. 2014;34(10):1033-1042. doi:10.1002/phar.1468

16. Berry TM, Prosser TR, Wilson K, Castro M. Asthma friendly pharmacies: A model to improve communication and collaboration among pharmacists, patients, and healthcare providers. J Urban Heal. 2011;88(SUPPL. 1):113-125. doi:10.1007/s11524-010-9514-9 
17. McBane SE, Dopp AL, Abe A, et al. Collaborative drug therapy management and comprehensive medication management - 2015. Pharmacotherapy. 2015;35(4):e39-e50. doi:10.1002/phar.1563

18. Yong YV, Shafie AA. How much does management of an asthmarelated event cost in a malaysian suburban hospital? Value Heal Reg Issues. 2018;15:6-11. doi:10.1016/j.vhri.2017.05.001

19. Crespo-Gonzalez C, Fernandez-Llimos F, Rotta I, Correr CJ, Benrimoj SI, Garcia-Cardenas V. Journal of the American pharmacists association characterization of pharmacists , interventions in asthma management : A systematic review. J Am Pharm Assoc. 2018;1-10. doi:10.1016/j.japh.2017.12.009

20. Portlock BJ, Holden M, Patel S. Original papers A community pharmacy asthma MUR project in hampshire and the isle of wight. Pharm J. 2009;282(January):109-112.

21. Bollmeier SG, Prosser TR. Community pharmacy-based asthma services : current perspectives and future directions. Integr Pharm Res Pract. 2014;3:49-70. doi:10.2147/IPRP.S47331

22. Garcia-Cardenas V, Armour C, Benrimoj SI, Martinez-Martinez F, Rotta I, Fernandez-Llimos F. Pharmacists' interventions on clinical asthma outcomes: A systematic review. Eur Respir J. 2016;47 (4):1134-1143. doi:10.1183/13993003.01497-2015

23. Adunlin G, Mahdavian S. The effectiveness of pharmacist interventions on asthma management: a systematic review. J Asthma Allergy Educ. 2012;3(6):264-273. doi:10.1177/2150129712464775

24. Mes MA, Katzer CB, Chan AHY, Wileman V, Taylor SJC, Horne R. Pharmacists and medication adherence in asthma: a systematic review and meta-analysis. Eur Respir J. 2018;52(2):1800485. doi:10.1183/13993003.00485-2018

25. Brown D, Portlock J, Rutter P. Review of services provided by pharmacies that promote healthy living. Int J Clin Pharm. 2012;34 (3):399-409. doi:10.1007/s11096-012-9634-2

26. Liberati A, Altman DG, Tetzlaff J, et al. The PRISMA statement for reporting systematic reviews and meta-analyses of studies that evaluate health care interventions: explanation and elaboration. PLoS Med. 2009;6(7):e1000100. doi:10.1371/journal.pmed.1000100

27. Effective Practice and Organisation of Care (EPOC). Suggested risk of bias criteria for EPOC reviews; 2016. Available from: http://epoc. cochrane.org/resources/epoc-resources-review-authors. Accessed November 25, 2018.

28. Khan KS, Kunz R, Kleijnen J, Antes G. Systematic Reviews to Support Evidence-Based Medicine. 2nd ed. London: Hodder \& Stoughton Ltd; 2011.

29. Verhagen AP, Ferreira ML. Forest plots. J Physiother. 2014;60 (3):170-173. doi:10.1016/j.jphys.2014.06.021

30. Fletcher $\mathrm{J}$. What is heterogeneity and is it important? $\mathrm{Br} \mathrm{Med} J$. 2007;334(7584):91-94. doi:10.1136/bmj.39038.614317.AE

31. Ried K. Interpreting and understanding meta-analysis graphs. A practical guide. Aust Fam Physician. 2006;35(8):1-24.

32. Israel H, Richter RR. A guide to understanding meta-analysis. $J$ Orthop Sport Phys Ther. 2011;41(7):496-504. doi:10.2519/ jospt.2011.3333

33. Stephenson J. Explaining the forest plot in meta-analyses. $J$ Wound Care. 2017;26(11):2017-2018. doi:10.12968/jowc.2017.26.11.611

34. Sedgwick P. How to read a forest plot in a meta-analysis. BMJ. 2012;351(December):h4028. doi:10.1136/bmj.h4028

35. Sedgwick P. Meta-analyses: heterogeneity and subgroup analysis. BMJ. 2013;346(7914):9-11. doi:10.1136/bmj.f4040

36. Zlowodzki M, Poolman RW, Kerkhoffs GM, Tornetta P, Bhandari M. How to interpret a meta-analysis and judge its value as a guide for clinical practice. Acta Orthop. 2007;78(5):598-609. doi:10.1080/ 17453670710014284

37. Hak T, van Rhee H, Suurmond R. How to interpret results of metaanalysis. (Version 1.3). 1st ed. Rotterdam, The Netherlands: Erasmus Rotterdam Institute of Management; 2016:1-21. doi:10.2139/ ssrn. 3241367
38. Chong-Ho Yu PD. Meta-Analysis and Effect Size. 2010:1-24. Available from: http://www.creative-wisdom.com/teaching/WBI/es. shtml. Creative Wisdom.

39. Faraone SV. Interpreting estimates of treatment effects: implications for managed care. PT J Formul Manag. 2008;33(12):700-711. doi:10.1093/jicru/ndm032

40. Durlak JA. How to select, calculate, and interpret effect sizes. $J$ Pediatr Psychol. 2009;34(9):917-928. doi:10.1093/jpepsy/jsp004

41. Fritz CO, Morris PE, Richler JJ. Effect size estimates: current use, calculations, and interpretation. J Exp Psychol Gen. 2012;141(1):218. doi:10.1037/a0024338

42. Charrois T, Newman S, Sin D, Senthilselvan A, Tsuyuki RT. Improving asthma symptom control in rural communities: the design of the better respiratory education and asthma treatment in hinton and edson study. Can Pharm J. 2006;139(4):44-50. doi:10.1016/j.cct.2004.07.004

43. Manfrin A, Tinelli M, Thomas T, Krska J. A cluster randomised control trial to evaluate the effectiveness and cost-effectiveness of the Italian medicines use review (I-MUR) for asthma patients. $B M C$ Health Serv Res. 2017;17(1):300. doi:10.1186/s12913-017-2245-9

44. Crowder T. An Evaluation of Community Pharmacists Applying the Patient Centered Care Approach to Ambulatory Oregon Health Plan Asthmatics in a Managed Care Setting. 2000. Oregon States University.

45. Bunting BA, Cranor CW. The asheville project: long-term clinical, humanistic, and economic outcomes of a community-based medication therapy management program for asthma. J Am Pharm Assoc. 2006;46(2):133-147. doi:10.1331/154434506776180658

46. Saini B, Krass I, Armour C. Development, implementation, and evaluation of a community pharmacy-based asthma care model. Ann Pharmacother. 2004;38(11):1954-1960. doi:10.1345/aph.1E045

47. Armour C, Reddel HK, LeMay KS, et al. Feasibility and effectiveness of an evidence-based asthma service in Australian community pharmacies: a pragmatic cluster randomized trial. J Asthma. 2013;50 (3):302-309. doi:10.3109/02770903.2012.754463

48. Bereznicki B, Peterson G, Jackson S, Haydn Walters E, Deboos I, Hintz P. Perceived feasibility of a community pharmacy-based asthma intervention: A qualitative follow-up study. J Clin Pharm Ther. 2011;36(3):348-355. doi:10.1111/j.1365-2710.2010.01187.x

49. Armour C, Bosnic-Anticevich S, Brillant M, et al. Pharmacy Asthma Care Program (PACP) improves outcomes for patients in the community. Thorax. 2007;62(6):496-502. doi:10.1136/thx.2006.064709

50. Cordina M, McElnay JC, Hughes CM. Assessment of a community pharmacy-based program for patients with asthma. Pharmacotherapy. 2001;21(10):1196-1203. doi:10.1592/phco.21.15.1196.33894

51. Mehuys E, Van Bortel L, De Bolle L, et al. Effectiveness of pharmacist intervention for asthma control improvement. Eur Respir J. 2008;31(4):790-799. doi:10.1183/09031936.00112007

52. Mangiapane S, Schulz M, Mühlig S, Ihle P, Schubert I, Waldmann HC. Community pharmacy-based pharmaceutical care for asthma patients. Ann Pharmacother. 2005;39(11):1817-1822. doi:10.1345/aph.1G180

53. McLean W, Gillis J, Waller R, The BC community pharmacy asthma study: a study of clinical, economic and holistic outcomes influenced by an asthma care protocol provided by specially trained community pharmacists in British Columbia. Can Respir J. 2003;10(4):195-202. doi: $10.1155 / 2003 / 736042$

54. Amirthalingam ARS. An evaluation of a community pharmacy based, pharmacist-led intervention package targeted to the patients' adherence status, to achieve and maintain target blood pressure (BP) control by optimising antihypertensive medicine adherence; 2017. Available from: http://etheses.bham.ac.uk/7834/. Accessed November 25, 2018.

55. Bereznicki BJ, Peterson GM, Jackson SL, Walters H, Fitzmaurice K, Gee P. Pharmacist-initiated general practitioner referral of patients with suboptimal asthma management. Pharm World Sci. 2008;30 (6):869-875. doi:10.1007/s11096-008-9242-3 
56. Schulz M, Verheyen F, Muhlig S, et al. Pharmaceutical care services for asthma patients: a controlled intervention study. J Clin Pharmacol. 2001;41(6):668-676. doi:10.1177/00912700122010438

57. Barbanel D, Eldridge S, Griffiths C. Can a self-management programme delivered by a community pharmacist improve asthma control? A randomised trial. Thorax. 2003;58(10):851-854. doi:10.1136/thorax.58.10.851

58. Bereznicki BJ, Peterson G, Jackson S, et al. Uptake and effectiveness of a community pharmacy intervention programme to improve asthma management. J Clin Pharm Ther. 2013;38(3):212-218. doi:10.1111/ jcpt.12017

59. Narhi U, Airaksinen M, Tanskanen P, Erlund H. Therapeutic outcomes monitoring by community pharmacists for improving clinical outcomes in asthma. $J$ Clin Pharm Ther. 2000;25(3):177-183. doi:10.1046/j.1365-2710.2000.00276.x

60. Saini B, LeMay K, Emmerton L, et al. Asthma disease managementAustralian pharmacists' interventions improve patients' asthma knowledge and this is sustained. Patient Educ Couns. 2011;83 (3):295-302. doi:10.1016/j.pec.2011.05.001
61. Grainger-Rousseau TJ, Mcelnay JC. Grainger-Rousseau,1997.pdf. J Appl Ther. 1997;1(1):145-161.

62. Herborg H, Soendergaard B, Froekjaer B, et al. Improving drug therapy for patients with asthma-part 1: patient outcomes. $J \mathrm{Am}$ Pharm Assoc. 2001;41(4):539-550. Available from: http://www. ncbi.nlm.nih.gov/pubmed/11486980

63. Bereznicki BJ, Peterson GM, Jackson SL, Walters EH, Fitzmaurice $\mathrm{KD}$, Gee PR. Data-mining of medication records to improve asthma management. Med J Aust. 2008;189(1):21-25.

64. Bereznicki B, Peterson G, Jackson S, Walters EH, Gee P. The sustainability of a community pharmacy intervention to improve the quality use of asthma medication. J Clin Pharm Ther. 2011;36(2):144-151. doi:10.1111/j.1365-2710.2010.01165.x

\section{Publish your work in this journal}

The Journal of Asthma and Allergy is an international, peer-reviewed open-access journal publishing original research, reports, editorials and commentaries on the following topics: Asthma; Pulmonary physiology; Asthma related clinical health; Clinical immunology and the immunological basis of disease; Pharmacological interventions and new therapies. The manuscript management system is completely online and includes a very quick and fair peer-review system, which is all easy to use. Visit http://www.dovepress.com/testimonials.php to read real quotes from published authors. 